\title{
Le droit d'initiative du sultan et les lois de Fâtih (Mehmed II)
}

\section{Halil Inalcık}

Traducteur : Faruk Bilici, Alexandra Koç et Pablo Moreno González

\section{(Q) OpenEdition Journals}

\section{Édition électronique}

URL : https://journals.openedition.org/ceb/839

DOI : $10.4000 /$ ceb.839

ISSN : 2261-4184

\section{Éditeur}

INALCO

\section{Édition imprimée}

Date de publication : 30 mars 2011

Pagination : 289-324

ISBN : 978-2-85831-189-7

ISSN : 0290-7402

\section{Référence électronique}

Halil Inalcık, "Le droit d'initiative du sultan et les lois de Fâtih (Mehmed II) », Cahiers balkaniques [En ligne], 38-39 | 2011, mis en ligne le 06 décembre 2011, consulté le 06 juillet 2021. URL : http:// journals.openedition.org/ceb/839; DOI : https://doi.org/10.4000/ceb.839

Ce document a été généré automatiquement le 6 juillet 2021.

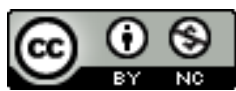

Cahiers balkaniques est mis à disposition selon les termes de la Licence Creative Commons Attribution - Pas d'Utilisation Commerciale 4.0 International. 


\title{
Le droit d'initiative du sultan et les lois de Fâtih (Mehmed II)
}

\author{
Halil Inalcık \\ Traduction : Faruk Bilici, Alexandra Koç et Pablo Moreno González
}

1 Vers la fin du IX siècle, les grands oulémas musulmans décrétèrent la fin de la libre interprétation (ictihâd) des écritures ${ }^{1}$. L'islam ne reconnaissait qu'une seule et unique loi : la charî'a $a^{2}$, qui dorénavant régissait la vie publique au même titre que les relations entre les individus, et ceci, en se basant sur des préceptes religieux. Un souverain musulman, qu'il soit calife ou sultan, ne pouvait agir en qualité de législateur. Celui-ci n'était que le garant et le dépositaire de la loi islamique, à savoir, la charî'a. S'il ne maitrisait pas les sciences religieuses au point de faire autorité en la matière, il ne lui était pas permis de pratiquer une interprétation personnelle de la charî̀a. Ces principes avaient pris place au sein de la jurisprudence islamique de sorte qu'ils devinrent irréfutables ${ }^{3}$. Par conséquent, l'Empire ottoman, en tant qu'État islamique, n'aurait dû avoir d'autre loi que la charî'a.

2 En réalité, l'État ottoman, ayant évolué dans des conditions tout à fait particulières, développa un ordre juridique qui s'étendait au-delà de la charî'a. Le principe qui le permettait était l'« örf», (ou coutume) qui, si on en donne une définition stricte, signifie l'autorité de légiférer dans les cadres en dehors de la charî'a en s'appuyant sur la seule volonté du souverain ${ }^{4}$. Cela put être obtenu moyennant l'acquisition par le souverain d'une assise absolue et centrale au sein de l'appareil étatique et par la prise en considération croissante de la primauté des intérêts de l'État sur toute autre question. Cette étape avait précisément été franchie par des États musulmans, notamment par des États turco-islamiques fondés à l'époque pré-ottomane. Le principe suivant lequel un système juridique distinct émanant de la seule volonté des souverains - à savoir, la loi et la coutume - coexiste avec la charî'a, était déjà amplement répandu chez ces États turco-islamiques lors de la période précédant l'arrivée des Ottomans. À présent, nous nous efforcerons d'expliquer de façon détaillée ce en quoi consiste l'örf, puis nous examinerons par la suite dans quelle mesure celui-ci est-il lié aux traditions de l'État turc. 


\section{Dimension de l'örf dans la jurisprudence islamique}

3 Il faut avant tout remarquer que l'örf dans le sens actuel d'us et coutumes employé en turc moderne, a été plus souvent désigné chez les Ottomans par l'expression örf-i ma'ruf

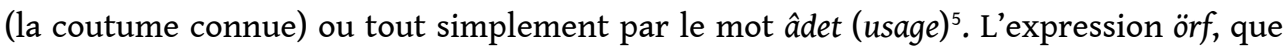
l'on rencontre parfois sous la forme d' örf-i sultânî (coutume sultanienne), désigne les lois établies par les souverains pour le bien de la communauté, relevant de ses seules prérogatives et donc, excluant la charî'a (lex principis) ${ }^{6}$. D’après Tursun Beğ, historien et administrateur de la Sublime Porte sous le règne de Fatih (Mehmed II), pour préserver l'ordre universel (nizâm-l âlem) et s'appuyant sur la raison, « la politique du Sultan et la Loi impériale sont définies par le terme d'örf selon nos savants'. " Les Ottomans réunissaient sous le même terme de ehl-i örf les personnes représentant de façon directe le pouvoir exécutif et l'autorité étatique. Seuls les ehl-i örf (forces de l'ordre), étaient habilitées à mettre en application les décisions du Cadı (juge). Ici l'expression " örf» comporte clairement les concepts d'autorité politique et de pouvoir exécutif.

Par ailleurs, les auteurs musulmans sont nombreux à avoir employé le terme örf dans le sens de âdet. Il existe néanmoins une relation étroite entre l'örf-i sultânî (loi sultanienne) et les örf-ü âdat (us et coutumes) : ainsi, le sultan s'étant octroyé le pouvoir d'établir des lois örfí (coutumières) en se basant directement sur son autorité, a pu, suivant le même mode, élever les örf-ü âdat à la catégorie de loi, par sa seule volonté. Autrement dit, la plupart des lois örfì étaient originellement des us et coutumes. En examinant attentivement ce dernier point on s'aperçoit que la volonté indépendante du sultan pour établir des lois constitue un élément essentiel de la production législative.

Une partie des oulémas, y compris Ibn Khaldûn, considérait illégitime l'existence d'un droit coutumier autre que la charî'a. Ils affirmaient que les quatre écoles juridiques ont donné à la loi islamique sa forme définitive ${ }^{8}$ et donc toute question touchant au droit est supposée être résolue. En revanche, pour d'autres, le principe de la coutume pouvait être appliqué aux questions étrangères à la charî'a ainsi qu'à certains cas reconnus par celle-ci comme étant situés à un niveau licite le permettant. Plusieurs d'entre eux allaient jusqu'à affirmer que la coutume représentait la cinquième source de la charî'a (sources de la jurisprudence islamique), après le Coran, la Tradition (la Sounna), le Consensus (l'idjma) et l'Analogie (la kıyâs) ${ }^{9}$. De même que certains fâkih, ou docteurs en jurisprudence, avaient déclaré préférer l'éventualité de privilégier l'örf par rapport aux règles obtenues par le procédé analogique ${ }^{10}$. Une décision relative à l'örf pouvait donc supplanter une autre décision résultant de l'Analogie. Cela dit, l'örf ne pouvait en aucun cas transgresser le Nâss (les textes fondamentaux: le Coran, le Hadith et le Consensus) ni le contredire. Ceux qui allaient encore plus loin englobaient l'örf dans le domaine du Consensus. Toutefois, on assimilait la coutume au Consensus dès lors qu'il s'agissait d'une règle qui était manifestement observée par l'ensemble de la communauté musulmane et approuvée par les oulémas, tant qu'elle n'entrait pas en contradiction avec le Coran et la Tradition. Certains d'entre eux avaient d'ailleurs tendance à légitimer toute tradition (ou usage) qui n'était pas contraire à la charî'a, tandis que d'autres considéraient qu'il fallait s'en tenir aux seuls cas de nécessité absolue ${ }^{11}$.

De manière générale, deux principes rendent la coutume licite selon les fâkih: la sauvegarde du bien et du "salut» de la communauté musulmane et le principe de justice $^{12}$. Ainsi, pour que la loi mongole puisse s'appliquer légalement aux peuples 
musulmans, le principe de justice a été invoqué, à savoir, le principe de protection des plus faibles par le pouvoir ${ }^{13}$.

7 En ce sens, il est du plus grand intérêt de s'attarder sur la fatwa (décret islamique) décrétée par Pir Mehmed ${ }^{14}$. Concernant la question de savoir si le raiyyet ${ }^{15}$ peut être rapatrié aux terres abandonnées par son père, celui-ci répond : « en réalité, cette question ne relève pas de la charî'a; cependant, il est communément admis que quiconque possède le mouton possède l'agneau. Or dans ce domaine il convient d'en référer aux ulü-l emr (le souverain), ou aux nombreux fonctionnaires mandatés par celles-ci, et dans tout les cas, de se plier à la volonté du souverain afin de préserver l'ordre public». On voit bien que les éléments de l'örf sont ici définis de manière on ne peut plus explicite :

- une situation non prévue par la charî'a

- l'existence d'une coutume répandue allant en ce sens ou tout simplement une considération d'ordre général qui servira de base à l'Analogie,

- la volonté du souverain (emr-i âlî, [l'ordre suprême]),

- la nécessité de recourir à l’örf pour des raisons d'ordre public.

\section{Les Turcs et le droit coutumier}

8 L'inauguration d'une ère nouvelle dans l'Histoire du droit musulman, marquée par l'importance croissante dont faisait l'objet la coutume, coïncida précisément avec la fondation des États turco-islamiques. Des savants comme Barthold, Becker, Gibb et Köprülü s'accordent à reconnaître qu'avec la fondation de ces États turco-islamiques, un changement essentiel se produisit tant au niveau de la perception de l'État islamique que dans le domaine du droit public. L'État acquit dès lors un caractère de suprématie absolue en tant qu'entité politique et exécutive au sein des organisations étatiques turco-islamiques - puis par la suite, mongoles -, par opposition à la conception traditionnelle de l'État islamique en tant que communauté politico-religieuse (umma). Le Droit coutumier finit par l'emporter en raison du fait que lui seul prenait en compte la primauté des intérêts et des besoins de l'État au-dessus de toute autre chose. Cette évolution donna une nouvelle dimension à la notion classique de califat. Le célèbre fragment ${ }^{16}$ de Râhat'us-Sudûr (1203) ${ }^{17}$ illustrait d'ailleurs la nouvelle situation de manière pour le moins éloquente : «La mission de l'imam est de s'occuper du sermon et de la prière... quant à l'exercice du pouvoir, il doit le remettre aux souverains et se résigner à lui confier la régence du profane ${ }^{18}$.

H.A.R. Gibb tente d'expliquer ce phénomène ${ }^{19}$ principalement par le renouveau de la tradition étatique de l'Iran ancien à l'époque de la domination turque. Nous pensons que le concept d'État turc y fut pour beaucoup. Or les Turcs qui pénétrèrent dans le monde musulman pour s'y établir en maîtres, venaient d'Asie centrale avec une vision ancienne et pérenne de l'État, ainsi qu'avec des traditions administratives préétablies. Cette conception du pouvoir se caractérisait par des usages et des croyances profondément enracinées, auxquelles la classe dirigeante, les bey et les groupes turcomans-oghouzes, restait attachée de façon obstinée ${ }^{20}$.

Ceci permet sans doute d'expliquer pourquoi, de nombreux États turcs, fondés de façon discontinue et éparse au fil des siècles - d'une part au Nord de la Chine, et d'autre part dans les régions du Nord de la mer Noire, et au Proche-Orient -, avaient malgré tout appliqué de manière constante, un certain nombre de principes organisationnels 
communs, et partagé les mêmes conceptions du pouvoir ainsi que les usages qui en découlaient, sans pour cela avoir été en contact les uns avec les autres ${ }^{21}$.

11 La nature des relations entre les sultans seldjoukides et le califat abbasside illustre parfaitement la place du souverain turco-musulman par rapport au concept de califat islamique. Lorsque Tuğrul Bey, le sultan seldjoukide, pénétra à Bagdad en 1055, ce dernier se vit confier l'autorité suprême de manière tout à fait officielle par le califat. Ses successeurs montrèrent ostensiblement qu'ils n'avaient pas l'intention de partager cette souveraineté avec qui que ce soit.

Le sultanat turc défendit sous couvert d'identité islamique le caractère absolutiste de son pouvoir en ne reconnaissant aucune autre autorité qui pût lui être égale ou supérieure. "Même les souverains turcs les plus soumis à l'Islam considéraient l'autorité de l'État comme supérieure à toute chose ${ }^{22}$ ».

13 Concernant la perception des États turcs primitifs, les inscriptions d'Orkhon représentent sans conteste une des sources les plus importantes dont nous disposons. Il transparaît à travers ces inscriptions que le khan, qui tire sa légitimité de Tanrı (Dieu), semble interpréter les idées de souveraineté et d'indépendance qui y sont évoquées dans le sens de pouvoir d'instaurer les lois: "Mes aïeux Bumin Hakan et Istemi Hakan furent intronisés pour régner sur les hommes, ce qui leur permit d'imposer et de régir la Loi en pays turc, tout en y habitant (Türk budunun ilin törüsin tuta birmiş, iti birmiş) ${ }^{23}$ ”. La tangibilité de l'État n'est rendue possible que par l'existence d'un khan législateur. Une fois que Temüdjin, ayant fondé un Empire à l'échelle mondiale - tout comme l'ont fait Mete ou Bümin Kagan - en s'appuyant sur les tribus turques et mongoles d'Asie centrale fut nommé Gengis Khan, c'est-à-dire, Empereur du monde, il promulgua instantanément la Yasa (La loi principale) (1206). Au moment de sa mort, celui-ci recommanda à ses descendants de ne s'écarter de la Yasa sous aucun prétexte, s'ils voulaient continuer à régner sur ce vaste Empire. Les dispositions de la Yasa ont cependant été complétées par de nouvelles lois (bilig) adoptées par les khans, toujours selon ce principe de légitimation par la seule volonté du souverain. Bien que cette loi puise certainement ses origines dans les usages et les principes étatiques des peuplades turco-mongoles des steppes d'Asie centrale (d'après la théorie de Barthold, Vladimirtsov et Z.V. Togan), Gengis Khan imposa la Yasa dans l'idée d'instituer à terme un nouvel ordre juridique des khans qui prévaudrait sur l'ancien, théorie soutenue avec pugnacité par Vernadski. D'après Z. V. Togan, depuis Mete jusqu'à Tamerlan, une loi traditionnelle perdura parmi les tribus turco-mongoles d'Asie centrale, dont les traits essentiels sont restés inchangés pendant des millénaires. Cette loi traditionnelle (Türe) ou loi principale (Yasa) structurait les bases de la souveraineté et de l'organisation étatique des khans, tout en permettant la coexistence de réglementations locales (mahalli kânunlar) régissant les interactions sociales des tribus subordonnées, à condition qu'elles ne contredisent pas le droit des khans. Toutefois, cette hypothèse semble refléter une bonne part de vérité. Même les descendants musulmans de Gengiz Khan continuèrent à observer scrupuleusement les principes fondamentaux de la Yasa surtout en matière de gestion étatique. Un exemplaire de la loi était religieusement conservé dans un coffret particulier par les descendants de Gengiz Khan qui régnaient en Iran ${ }^{24}$. Al-Maqrizi ${ }^{25}$ exprima sa conviction que Baraka, khan musulman de la Horde d'Or, assura la primauté de la Yasa dans les domaines relatifs à la souveraineté étatique grâce à l'influence significative qu'il exerçait sur l'Égypte et la Syrie. La concurrence entre la Yasa et la charî'a au Proche-Orient s'est à l'époque traduite par une 
concurrence accrue entre beys et oulémas. On a d'ailleurs soupçonné les oulémas fondamentalistes ${ }^{26}$ d'avoir joué un rôle dans l'assassinat d'Ulugh Beg, le petit-fils de Tamerlan, qui était très attaché à la loi ${ }^{27}$. Malgré la vive résurgence de la charî'a qui se généralisa pendant les $\mathrm{XIV}^{\mathrm{e}}$ et $\mathrm{XV}^{\mathrm{e}}$ siècles (il convient de se remémorer à ce titre l'exemple du Kadı Burhaneddin ${ }^{28}$ ), le modèle de l'État ilkhanide exerça en Anatolie une influence significative et constante en matière juridique et administrative, en particulier sur les principautés turcomanes des marches frontalières des débuts de l'Empire Mongol (y compris ottoman), qui demeuraient profondément attachées à leurs traditions centre asiatiques. Le poète Ahmedî, né vers la fin du XIVe siècle dans la région de Germiyan, située aux confins de l'Empire, louait par ses célèbres vers panégyriques l'administration mongole et la Yasa ${ }^{29}$. Il convient d'ailleurs de souligner que le principe de justice y est évoqué - comme on pouvait l'observer dans les poèmes d'Al Maqrizi - en tant qu'élément de légitimation de la primauté de la Yasa

Les lois que les souverains promulguèrent par leur seule initiative furent rassemblées sous le nom de Yasa ou Yasaknâme (Code juridique) après le règne des Ilkhanides, aussi bien dans l'Empire ottoman, que dans les États turcomans qui se formaient en Asie centrale et en Iran (tels que les Ak koyunlu ou les Moutons noirs par exemple). De même que dans le Tüzüukât-i Temur (Code juridique de Tamerlan) l'esprit de la Yasa y est omniprésent. Dans tous ces États, l'existence de deux institutions distinctes illustre de la façon la plus évidente la stricte séparation entre le droit coutumier (örfi) et la charî'a: le kadıaskerlik (kedıleşkerlik), tribunaux chargés de statuer sur des affaires concernant les domaines administratifs et militaires et le yargucilik, [ou tribunaux civils], chargés de statuer sur les questions liées au domaine civil, tout en limitant les prérogatives de la charî'a. Dans la région du Khwarezm, en Iran et en Irak, des tribunaux de juges coutumiers se juxtaposèrent aux tribunaux de juges religieux. Les kadıaskerlı (tribunaux militaires) existaient déjà en Anatolie seldjoukide. On sait désormais grâce à des inscriptions retrouvées dans une mosquée de Bagdad, que l'autorité juridique avait été séparée de la juridiction religieuse en $1357^{30}$.

Sous les Ottomans, le kadiaskerlik désignait la fonction des membres de l'État. Les infractions commises par les militaires, le partage des successions, etc., n'étaient pas jugées par les tribunaux de la charî'a, mais par les kadiasker. La séparation entre le Droit public et la charî'a était manifestement respectée par l'État ottoman - et parfois même dans ses détails les plus subtils -, dans le prolongement de la tradition que nous avons tenté d'expliquer ci-dessus. Nous allons ci-après essayer de le démontrer, puis nous passerons en revue l'évolution du droit ottoman jusqu'à l'époque de Mehmed II.

\section{Le Droit coutumier jusqu'à l'époque de Mehmed II}

Même si on rapporte ${ }^{31}$ qu'à l'époque d'Osman Gazi certaines lois relatives au système des finances publiques et du timar (fermage) avaient été rédigées, il n'est pas nécessaire de dire que cette affirmation n'a aucune valeur. Il y a cependant un point que nous pouvons affirmer avec certitude : Osman se trouvait, à partir de 1310, à la tête d'un organe politique structuré, le beylik ${ }^{32}$. Une autre nouveauté, qu'il convient d'attribuer à l'époque d'Osman Gazi, concernant l'incompatibilité notoire de la charî'a et de la coutume. À la suite d'un fait relatif à la levée d'un impôt sur les transactions commerciales, Osman Gazi formula la question suivante : "'Est-ce un ordre de Dieu ou bien est-ce une initiative des beys ?' Une personne répliqua alors: 'C'est la tradition, 
Excellence, elle l'est depuis des temps immémoriaux.' Là-dessus Osman Gazi le réprimanda. 'Mais le commerce est une habitude propre aux beys!', protestèrent certains. C'est alors que le souverain acquiesça et ordonna la mise en place de cet impôt, puisqu'il s'agissait d'une coutume ${ }^{33}$.»

17 À l'époque d'Orhan Bey ${ }^{34}$, l'Anatolie centrale se trouvait toujours sous le joug Ilkhanide, et le pays dont était issu Orhan comptait parmi les vilayet (provinces) des marches frontalières de l'Empire (Ucât) soumis au paiement de taxes alimentant la trésorerie des administrateurs ${ }^{35}$. Selon $\mathrm{Z}$. V. Togan, « Le seul ordre étatique et juridique que reconnaît Orhan Bey est celui qui est incarné en l'espèce par la Loi traditionnelle (Türe) et la Loi principale (Yasa). La quintessence de l'État ne réside non pas dans la charî'a, mais dans la $\mathrm{Yasa}^{36}$ ». Au vu des registres ${ }^{37}$ des fondations impériales (vakıf) datant du XVI ${ }^{e}$ siècle du sandjak de Hüdâvendigâr (Bursa et sa région) dans lesquels figurent les différents actes de cession et de mainmorte consignés à l'époque d'Orhan, il est évident que $\mathrm{Z}$. V. Togan parvient à des affirmations péremptoires à ce sujet. Il existait des individus chargés de gérer le dispositif administratif centralisé qu'a développé de manière significative Orkhan Bey, assorti de documents conformes aux préceptes de la charî'a. Les premiers vizirs étaient issus de la classe des oulémas. D'après le contenu des actes de cession et de mainmorte relatifs à cette période, il en ressort que les bases fiscales existaient déjà à cette époque.

En 1366, Murad Bey ${ }^{38}$ accorda le privilège ${ }^{39}$ de la charge d'une fondation pieuse à Ahi Musa, à qui il garantit « [la totale exemption] du paiement des taxes correspondantes : avâriz-i divvanîlye] (contributions exceptionnelles levées notamment en temps de guerre) tekâlif-i örfî (taxes décrétées par le souverain ou impôts arbitraires), salgun ${ }^{40}$ (tribut annuel imposé à une communauté), tout en étant exempté des charges suivantes : messager, segban (dresseur des chiens de chasse du sultan) cerahor ${ }^{41}$ (ouvrier dans les garnisons), et nâib (substitut du cadi) ${ }^{42}$ ”. Les types de fonctions et d'impôts énumérés ci-contre, relèvent complètement de l'örf, c'est-à-dire qu'ils reposent sur des règles coutumières. Il convient également de signaler que ce document et autres titres de propriétés dans lesquels sont énumérées ces exemptions, montrent tant par leur forme que par leur contenu, que ces pratiques sont liées aux anciennes institutions turco-mongoles Tarhanides ${ }^{43}$.

À l'époque de Beyazîd I ${ }^{\text {er }}$ (1389-1402) fut fondée la première administration ottomane centralisée, s'étendant du Taurus au Danube. Même si à cette période, le sultan ottoman apparaît dans les sources arabes sous la qualification de "Sâhibu'l-Ucât" (Maître des marches frontalières de l'Empire), c'est en tant que maître d'un grand Empire musulman qu'il incarne une nouvelle étape en terme de production juridique et d'organisation des lois. Un vieux récit ottoman ${ }^{44}$ relatant la période de Beyazîd, la décrit comme étant une période caractérisée par l'introduction d'un certain nombre de nouveautés telles que la consolidation d'un Trésor étatique désormais centralisé, la mise en place de nouveaux impôts, l'introduction du système de registres et de consignation d'archives, la réforme des fonctions de cadi, etc. De cette façon, les prétentions de la charî'a mises à mal, celle-ci représentera un soutien à toutes sortes de mécontentement vis-à-vis du souverain. En revanche, le célèbre âlim (savant) ottoman Mehmed Fenârî, dont les oulémas d'Égypte mentionnaient le nom avec grande déférence, exerçait à l'époque, en qualité de représentant de la charî'a, une grande influence dans les affaires de l'État. En outre, c'était un contemporain du célèbre âlim en Jurisprudence islamique, réfractaire à l'örf, şeyh Bedreddin Mahmud - dont l'œuvre Câmi'ul Fusûleyn a été une référence durant des siècles. Le récit qui témoigne de l'opposition de Mehmed 
Fenârî à l'encontre de la tentative de Beyazid "La foudre », de prendre une décision de manière unilatérale concernant une question juridique, se doit d'être nuancé ${ }^{45}$. Par ailleurs, nous sommes en mesure d'attester avec certitude des activités de Bâyezid concernant l'adoption de règlements et l'introduction de nouvelles normes juridiques grâce à des documents officiels émis jusqu'à cette époque. À titre d'exemple, les archives les plus anciennes consignées grâce au système des registres basé sur l'Administration centrale ottomane, le droit fiscal sultanien et le droit foncier datent de la régence de Bâyezid. La profonde réforme de l'administration chargée de la levée des esclaves, instituée par des règlements coutumiers, est également attribuée à cette période ${ }^{46}$. Dans l'optique de fonder son système d'administration militaire, Bâyezid, comme le fera Mehmed II par la suite, n'a pas hésité à adopter à maintes reprises des mesures affectant les oulémas, en s'emparant au nom de l'État d'un certain nombre de vakfs dont bénéficiaient les représentants de la charî'a pour les mettre à la disposition de la classe militaire. Les hauts intérêts de l'État de même que les nécessités d'ordre militaire ont toujours été le souci premier des souverains.

Lors de l'interrègne (à partir de 1402), le morcellement de l'Empire ottoman de même que les conflits internes qui l'affectaient ont naturellement entravé les améliorations initiées à la période précédente. L'interrègne ne prend pas vraiment fin en 1413, mais s'étend plutôt jusqu'en 1422, date à laquelle Düzme Mustafa ${ }^{47}$ tomba en disgrâce. En un sens, le mouvement mené par şeyh Bedreddin Mahmud $^{48}$ en 1416, est le signe manifeste d'un mécontentement ressenti dans les provinces militaires des marches frontalières et parmi les nomades Turkmènes, face à un pouvoir central dont le caractère orthodoxe et "étatiste» tend à se renforcer toujours plus ${ }^{49}$. La répression étatique face à ce soulèvement, a permis aux représentants de la charî'a de gagner en influence au sein de l'administration, voire, est une conséquence directe de la levée d'une partie des mesures prises à leur encontre sous Bâyezid Ier. Les prérogatives de l'État ont dû, d'une manière ou d'une autre, s'affaiblir. Ceci se devine aisément au vu des concessions faites aux familles des Beys locaux, et par la politique sommes toutes désinvolte de l'État en matière d'administration des terres et des vakfs.

21 L'abondance de documents relatifs au règne de Murad II (1421-1451), atteste que le droit coutumier s'est alors solidement intégré au sein de l'organisation étatique selon des modalités classiques. Parmi ces documents, se trouve le registre des $\operatorname{timar}^{50}$ de la province d'Albanie ${ }^{51}$ daté de 1431 - sans doute le plus riche en détail -, qui révèle que le système des timar, les taxes imposées par le souverain ottoman, le statut des civils et de la classe militaire, ont perduré dans leur globalité tout comme dans leurs moindres détails, jusqu'au XVI e siècle, comme on l'a vu, en évoquant brièvement les coutumes ainsi que les bases de l'organisation étatique relatives à cette période ${ }^{52}$. En fait, le Kanûnnâme (règlement organique) de Mehmed II allait véritablement prendre corps une fois qu'il y aurait adjoint les règlements antérieurs à 1451

\section{La place de Mehmed II dans l'évolution du droit}

Le fait que le règne de Mehmed II marque un tournant dans l'évolution du droit ottoman a déjà été systématiquement signalé par les personnes ayant écrit sur le sujet, toutefois ils ne se sont pas assez attardés sur la question de sa véritable nature. L'hypothèse que certains avancent, selon laquelle le droit ottoman et son organisation 
seraient un produit du système byzantin est sans fondement (nous nous contenterons à ce propos de conseiller au lecteur les analyses de Fuat Köprülü, plus fiables ${ }^{53}$ ).

L'évolution du droit à l'époque de Mehmed II repose sur un facteur fondamental : le fait que, suite à la prise de Constantinople, celui-ci ait gagné une autorité illimitée, et qu'il ait fondé ad vitam eternam un Empire absolu et centralisés ${ }^{54}$. Istanbul a permis à Mehmed II de réaliser le dessein d'asseoir concrètement son pouvoir absolu, à travers les nouveautés qu'il a introduites tant sur le plan juridique qu'au niveau de l'organisation étatique, et grâce à cette autorité, il a érigé le droit coutumier au rang de droit suprême.

24 Aussi autant qu'il est le véritable fondateur de l'Empire, Mehmed II est incontestablement le souverain ottoman ayant le plus marqué la conception du droit ottoman et de ses lois, et ce, de manière ininterrompue et irréversible. On peut simplement considérer que, l'audace qu'il a eue de franchir cette étape par rapport à ses prédécesseurs, résultait simplement du fait qu'il était devenu puissant au point de pouvoir être l'instigateur de cette rupture fondamentale avec l'ordre établi. Mehmed II était le souverain ottoman le plus influent et le plus libre dans ses idées, ce qui lui a permis d'employer ses prérogatives de souveraineté absolue, afin de s'assurer une grande liberté d'action dans l'établissement des lois et la mise en place d'un État répondant à des critères bien précis. Afin de pouvoir asseoir son autorité absolue au sein de l'État, il s'est préalablement débarrassé du tout puissant Vizir Çandarlı Halil Pacha, qui était de lignée noble, puis exception faite d'une personne, il remplaça l'intégralité de son vizirat par des individus qu'il avait choisis parmi les esclaves. Quant au Grand Vizir Mehmed Pacha de Karaman (1477-1481) qui appartenait à la classe des oulémas, il a été un des acteurs ayant contribué le plus fortement au renforcement du droit coutumier, et par conséquent de l'autorité du souverain. Le grand savant Kemal Paşa zâde, un contemporain de Mehmed II, met en évidence l'importance majeure que représente pour l'establishment le système de levée d'esclaves, lui permettant d'asseoir une autorité étatique toujours plus centralisée $e^{55}$.

\section{Les règlements de Mehmed II}

Outre les différents règlements qu'il a promulgués sous forme de décrets, Mehmed II a adopté deux kanûnnâme $e^{56}$ additionnels. Ceux-ci constituent sans aucun doute une nouveauté remarquable, tant au niveau de la forme que du contenu, en comparaison avec la théorie juridique islamique. Le fait que le souverain, par sa seule volonté, adopte des lois pour le bien de l'État, indépendamment de la charî'a, avec laquelle elles peuvent parfois rentrer en conflit, est à associer non pas aux fondements islamiques, comme on a tenté de l'expliquer plus haut, mais à la tradition turco-mongole. Les sources mentionnent l'existence d'une hiérarchisation des règlements organiques, dans les États islamiques antérieurs à Mehmed II, notamment sous l'Empire Seldjoukide à l'époque de Malikşah ${ }^{57}$ ou des Ilkhanides ${ }^{58}$.

Les deux kanûnnâme adoptés par Fatih, le premier sur l'organisation de l'État ${ }^{59}$ et le second dans le domaine administratif, financier et pénal se présentent sous forme d'une compilation de règlements officiels organisés en chapitres et sous-chapitres, dans lesquels ces règlements sont, dans la mesure du possible, classés selon un ordre systématique. Cette nouveauté ${ }^{00}$ laisse à penser qu'il a en fin de compte existé un recueil officieux de kanûnnâme régissant les protocoles officiels et encadrant les 
pouvoirs des cadis. Toutefois, celui-ci ne présentait pas les caractéristiques d'un code public. Il s'agissait en fait d'une esquisse juridique schématisée «ayant pour but de donner au sultan et à son personnel administratif un aperçu global sur l'organisation de l'Empire et ses institutions ». Nous avons la conviction que bien qu'une partie des règlements organiques réponde à cette description, une autre partie des kanûnnâme, à l'instar des législations de Mehmed II ou de Soliman le Magnifique possède un caractère officiel. Pour corroborer notre idée, nous pouvons ici citer les points suivants : dans un kanûnnâme ${ }^{61}$ du Palais de Topkapı, il est fait mention d'un règlement organique en possession du Beylerbey d'Anatolie que celui-ci avait envoyé au Nişancı (Chef de la Chancellerie impériale) d'Istanbul, lequel, après l'avoir confronté avec le nouveau règlement organique (il est peu probable qu'il s'agisse d'une loi propre à chaque sandjak $k^{62}$ ) en possession du Divan (Conseil impérial), annotait les différences en marge du document, et attestait par écrit qu'il avait bien remis ce présent document au pacha en y joignant le paragraphe suivant: "Que nos très estimés pachas fassent appliquer la sentence dont il est question ici conformément au kanûn-i şerif (Loi sacrée) . ${ }^{63}$ Les formulations "règlement organique récemment entré en vigueur ${ }^{64}$ " apparaissant dans un certain nombre de règlements organiques parvenant jusqu'à nous, montrent qu'il existait des compilations de lois, dont la validité se limitait à une période déterminée. Enfin, Ahmed I Ir65, dans son adaletnâme ${ }^{66}$ (Édit de Justice) de 1596, met clairement en évidence les pratiques de Kanûni Süleyman ${ }^{67}$ à ce sujet : aussitôt qu'il adoptait une loi, il le faisait savoir dans toutes les villes et dans tous les tribunaux de cadi par le biais d'une circulaire officielle, afin que celle-ci soit immédiatement appliquée. Enfin, nous aborderons ensuite les indices relevés dans les kanûnnâme de Mehmed II et de Süleyman, qui sont autant de preuves confirmant notre opinion.

\section{La loi sur l'organisation}

27 Le règlement organique de Mehmed II relatif à l'organisation étatique résulte d'un ensemble de règlements rassemblé à son initiative, et a été promulgué après avoir été soumis à son contrôle et complété par ses soins (L'exemplaire qui est en notre possession nous est parvenu assorti des amendements qui y ont été rajoutés en 1488, sous Bâyezid II. Par exemple, les deux pour cent prélevés sur les biens importés d'Europe, au titre de la taxe douanière est une initiative qui émane du Sultan Bâyezid $\mathrm{II}^{68}$. Vers la fin du règne de Mehmed II, cette taxe s'élevait à cinq pour cent. En fait, les modifications opérées sur le texte original de Mehmed II sont en général du même ordre, c'est-à-dire qu'elles concernent des détails insignifiants). Le souverain, dans son Kanûnnâme relatif à l'organisation étatique, ordonna à son chancelier Leys-zâde de réformer un règlement qui était sur le point d'être promulgué en sa présence, au divan. Ce dernier rassembla sur-le-champ des règlements antérieurs au règne de Mehmed II, en vigueur à l'époque de ses ancêtres, que le Sultan s'empressa d'examiner, en comblant lui-même les lacunes du texte ainsi obtenu. Le chancelier a ensuite indiqué que ce règlement organique était le résultat de la transposition fidèle des propos du sultan. En tête de ce Kanûnnâme, figure une ligne écrite de la main du sultan, qui est en fait un ordre émanant directement de sa personne qu'il formule en ces termes: "Ce kanûnnâme est la loi de mon père et celle de mes aïeux ; cette loi est aussi la mienne et sera celle de mon auguste descendance qui sera tenue d'y demeurer fidèle, génération après génération. "

Ce kanûnnâme est divisé en trois parties. 

vraisemblablement hiérarchisées après le court vizirat du Nişancı Mehmed Paşa de Karaman (1477-1481) ${ }^{71}$. sultan au Nişancı Leys-zâde Mehmed fils de Mustafa. D'un certain point de vue, le Nişancl est à l'örf ce que le Mufti ${ }^{72}$ est à la charî'a (aussi surnomme-t-on le Nişancl "müfti$i$ kanûn"(le Moufti de la Loi)). Tous les règlements produits à l'initiative du souverain passaient par le Nişancl, puis leur confirmation, c'est-à-dire l'apposition du monogramme sultanien, était également effectuée par son intermédiaire. Par ailleurs, celui-ci veillait scrupuleusement à la bonne tenue des registres (se présentant sous forme de livres des comptes), dans lesquels étaient recensés le système fiscal, l'organisation des timars et la gestion des biens fonciers de l'Empire. D'ailleurs les règlements et décrets relevant du droit coutumier, dont la primauté s'appliquait à ces domaines, étaient la plupart du temps consignés dans ces registres. Ainsi, les derniers textes de loi récemment ratifiés se trouvaient en possession du Nișancl. Au sujet des affaires relevant du droit coutumier, c'est d'ailleurs lui qui était chargé d'annoncer en dernier ressort l'entrée en vigueur de la nouvelle Loi, outre le fait qu'il était nécessaire de le consulter préalablement ${ }^{73}$ avant promulgation et entrée en vigueur définitive. C'est probablement pour ces raisons que Fatih a transféré la tâche de la compilation des lois au nişancl. Nous verrons de fait que la disposition des règlements sultaniens était généralement effectuée par le Nişanct.

Ce kanûnnâme, dont les règles concernent directement la gestion de l'État sont à inclure dans les règlements coutumiers. Dans le texte ${ }^{74}$, Mehmed II indique en personne qu'il a "[ordonné] ces lois sultaniennes ${ }^{75}$ \%. Le mode d'organisation gouvernemental, sa composition, l'étendue de ses pouvoirs, la nature de ses relations avec le souverain, les différents rangs et grades de ses membres, les titres honorifiques, les appointements et les pensions de retraite, les condamnations, sont des questions qui sont toutes définies, non pas par la charî̀a, mais par la seule volonté du Sultan. Le fait que soient mentionnés dans le Kanûnnâme les possessions et services relatifs au Palais, qui apparaissent donc parmi les questions d'organisation étatique, relève d'une particularité propre à l'administration ottomane ; car le gouvernement et le Palais sont considérés comme deux entités complémentaires formant un tout indissociable. À l'intérieur du palais du sultan, le personnel du Enderûn ${ }^{76}$ et le personnel aux portes du Enderûn, sont perçus comme étant de même nature, et les individus affectés au service du Enderûn sont amenés à assumer des fonctions au sein de l'appareil gouvernemental (système des $\mathrm{Kul}^{77}$ ). D'autre part, il a été démontré que les ouléma, en tant que serviteurs de l'État, avaient également une place et un rang au sein de l'organisation étatique.

Les lois rassemblées au sein du droit coutumier que nous allons examiner plus en détail ci-après sont généralement produites sous forme de firmans ${ }^{78}$ impériaux. Ceci en raison du fait que la source et le fondement de ces lois émanent de la volonté du sultan. Cellesci consistent en un ordre donné par chacun des Sultans, et comportent des éléments spécifiques, que l'on retrouve généralement dans les firmans impériaux. Ce qui explique que le décret impérial se soit imposé en tête du Kanûnnâme. Ceci confirme donc qu'il s'agit bien d'un texte de loi faisant autorité. Par conséquent, les articles du Kanûnnâme prennent la forme de règles ayant force obligatoire (ma'mûlün bih) par le simple fait que le souverain les décrète comme tels.

Cahiers balkaniques, 38-39 | 2011 

afin que la force obligatoire de la loi acquière un caractère intemporel ${ }^{79}$. Or, en droit ottoman, les décisions prises par un sultan pendant la durée de son règne, à savoir, les lois, les alliances que celui-ci ait pu conclure de même que les distinctions ou titres honorifiques que celui-ci ait pu accorder, n'impliquent plus ses successeurs, qui ne sont pas tenus de les maintenir. Afin que ces décisions conservent leur force obligatoire, il est donc nécessaire que le nouveau souverain les confirme à nouveau. Mehmed II, affirme que les lois du Kanûnnâme émanent de son père et de son grand-père, et par la formulation "Cette loi est aussi ma loi »", il remplit les conditions de cette nécessaire validation des dites lois. Toutefois, il souhaite en outre soumettre ses successeurs à ces règles de Droit et il ne fait aucun doute qu'en agissant ainsi, il s'octroie une position particulière au sein du Droit en qualité de dépositaire de la Loi. Ceci s'explique aisément étant donné qu'Istanbul entretenait le mythe selon lequel Mehmed II était le véritable fondateur de l'Empire. Cette conception du souverain apparaît dans la déclaration du Nişancı en préambule du Kanûnnâme. Selon le Nişancı la nécessité que Mehmed II donne naissance à un Kanûnnâme en rassemblant les lois de ses ancêtres tout en les complétant, s'était imposée comme une évidence, au vu des innombrables conquêtes à son actif, surtout quand il s'agit d'une victoire aussi importante que la conquête d'Istanbul. Il convient de se rappeler qu'en ces lieux, des lois et coutumes furent proclamées lois éternelles par les Khan qui y avaient fondé leur empire.

l ne fait aucun doute qu'une grande partie de ces lois et de ce système juridique, comme il était spécifié dans les décrets impériaux, est antérieure à Mehmed II. De même que les formules protocolaires "Ceci est ma Loi » ou bien "j'en ai donné l'ordre " que celui-ci utilise, montrent bien qu'il n'a rien fait d'autre que de confirmer à maintes reprises des coutumes et règles préexistantes. Cependant, lorsqu'il affirme que "les nominations du Defterdârllk ${ }^{81} d u$ Nişancllk $^{82} y$ compris celle des professeurs des medrese, relèvent de son autorité ", il est clair qu'il s'agit d'une production juridique qui émane de celui-ci, dans la mesure où nous savons que les medrese Sahn ${ }^{83}$ ont été créées à l'initiative du Sultan Mehmed II. On sait grâce à d'autres sources que la création d'un Arz oda ${ }^{84}$ réservé aux Hauts dignitaires du gouvernement leur permettant de recevoir pour audience en représentation du sultan, est aussi une autre de ses initiatives. Par ailleurs, il annonce explicitement la modification d'une règle de la manière suivante : « La loi sur les individus composant la suite autorisée à partager mes repas n'émane pas de mon auguste personne, mais de la décision de mes ancêtres. Mon illustre grand-père avait pour habitude de partager ses repas avec ses ministres. J'ai pris la décision de l'abroger $»^{85}$. Les autres modifications qu'il a apportées concernaient probablement des protocoles, la réorganisation de la hiérarchie, le montant des pensions et salaires. Toutefois, nous sommes en mesure d'attester la mention dans le Kânûnname de la modification des affectations des fonctionnaires, du personnel de service (hizmetler) et des règlements par le biais des registres officiels, seulement à partir de Murad II ${ }^{86}$.

De ce point de vue, il est intéressant d'examiner le chapitre relatif au fratricide. Beyazid $\mathrm{I}^{\mathrm{er}}$, avait exécuté son frère Yakub sur le champ de bataille lors de la guerre du Kosovo (1389 avec l'assentiment de ses vizirs dans le but de prévenir une guerre civile. Par la suite, la guerre civile à l'origine de la division de ses fils a ébranlé l'État au point de devoir à nouveau légaliser le fratricide. Lorsque Mehmed $\mathrm{I}^{\mathrm{er}}$ avait désigné son fils aîné comme héritier du trône, il avait pris des mesures préalables afin de sauver ses deux fils cadets d'une mort certaine. Murad II, avait emprisonné son frère Mustafa, qui

Cahiers balkaniques, 38-39 | 2011 
s'était rebellé contre lui en s'unissant à Byzance et aux fils de l'émirat de Karaman ${ }^{87}$, puis le fit exécuter. Se référant à ces événements, Dukas ${ }^{88}$ souligne le fait que le fratricide constitue chez les Ottomans une tradition. À peine Mehmed le Conquérant accéda-t-il au Trône, qu'il se débarrassa de son jeune frère Ahmed, comme l'avaient fait avant lui ses ancêtres lors de leur accession au pouvoir. En témoigne un ancien règlement de son Kanûnnâme dans lequel il y fait référence de la manière suivante :

"Occire les membres de sa fratrie est une nécessité si on poursuit le dessein de maintenir l'ordre au sein de l'Empire, ainsi que le permettent la plupart des ouléma ${ }^{89}$ dans le cadre de la Loi Islamique ».

destion des cadis ${ }^{91}$, mentionné dans ce Kanûnnâme, dans un autre firman impérial datant du mois de Receb de 884 année hégirienne (Septembre-Octobre 1479). Dans le Kanûnnâme, ce chapitre se présente de la manière suivante: «Les cadis devront percevoir sept aspres pour la gestion des registres, trente aspres pour l'émission de cadastres, douze aspres pour l'émission d'une copie de registre ; en ce qui concerne la gestion des dotes, ils percevront vingt aspres par tranche de mille et trente-deux aspres pour les contrats de mariage si la jeune mariée est vierge, et quinze aspres si celle-ci est veuve. » À présent examinons la façon dont est formulée la même question dans le firman évoqué :

"[...] Qu'il soit rendu public, que sous leur forme actuelle, les questions de la demande en mariage (bab-i kismet), de la validation de l'union matrimoniale (husus-i nikah), ainsi que les questions relatives à l'affranchissement ou l'affranchissement des esclaves (itakname), ayant fait l'objet de correspondances officielles dans lesquelles il est fait état de penchants disproportionnés des cadis pour l'une ou l'autre des parties, et dont l'impartialité excessive a donné lieu à des pratiques injustes engendrant un certain nombre de litiges, j'ai par conséquent ordonné par ce firman une loi énumérant les principes suivants: qu'il soit attribué au cadi citulaire trente aspres par décision de libération ou d'affranchissement, ainsi qu'un aspre chacun pour le substitut du cadi et son secrétaire, quant aux courriers, exception faite des itakname [lettre d'affranchissement], le cadi titulaire percevra quinze aspres de même que son substitut et son secrétaire percevront un aspre chacun; pour les contrats de mariage, le cadi titulaire percevra vingt aspres, et son substitut percevra la même quantité, si la future mariée est vierge; concernant les contrats de mariage impliquant une veuve, le cadi titulaire percevra moins de vingt aspres et vingt aspres par tranche de mille pour couvrir les frais administratifs... ".

Cet exemple mérite attention dans la mesure où il permet de mettre en exergue la corrélation entre les sources des différentes lois organiques et les Firmans impériaux que nous évoquerons ci-dessous.

Si on rassemble tous les chapitres relatifs au protocole ainsi que les noms et titres mentionnés à la fin du Kânûnname, on constate que ces derniers confèrent à la loi organique des allures recueil de protocole. Cela dit, il convient de ne pas oublier que sous l'Empire ottoman, le protocole et l'organisation de l'État forment un tout indissociable. 
40 Le premier chapitre consacré aux dignitaires de l'État, se clôt de la manière suivante : "L'organisation de l'Etat a été réformée de cette manière, que désormais ma noble descendance contribue à son amélioration ». Le fait que Mehmed II reconnaisse que les lois qu'il avait lui-même promulguées puissent être améliorées représente une vision juridique qu'il ne faut pas négliger.

41 Seulement une partie des ordonnances et règles édictées dans le droit administratif a été incorporée au Kanûnnâme de Mehmed II ${ }^{92}$. La seconde loi organique du souverain concerne le droit civil (reâyâ kanûnu). C'est-à-dire que le système fiscal rend compte de la nature des impôts que versent les sujets ottomans au souverain. De cette façon, le fait que les Kânûnname soit séparés en deux catégories, à savoir, la catégorie relevant du domaine militaire et l'autre du domaine civil, reflète une conception particulière $d u$ pouvoir de la part de l'État ottoman tant au niveau de sa structure que de sa constitution: ainsi appartiennent à la classe militaire toutes les catégories de personnes travaillant au service de l'État et percevant un salaire de celui-ci. Ceux-ci sont soumis à l'autorité du kadiasker ${ }^{93}$. La catégorie des civils (reâya) englobe tous les sujets ottomans n'appartenant pas à la classe militaire (askerî şümûlu) et qui paient l'impôt.

\section{Le règlement de droit civil}

42 Ce second Kânûnname de Mehmed le Conquérant nous est connu grâce à une copie qui a été faite à l'époque de Beyazid II. Cette copie date de la fin du cemaziye'el-âhir de $893 \mathrm{~h}$. (2-10 juin 1488) et a été exhumée par Fr. Kraelitz ${ }^{94}$. Certains indices permettent d'affirmer que ce Kânûnname a été selon toutes vraisemblances, produit immédiatement après la prise de Constantinople en1453. En premier lieu, la référence essentiellement utilisée pour mesurer la récolte était le boisseau d'Andrinople (Edirne müddü), correspondant à deux poignées de grains. Deuxièmement, ceux qui passaient par les territoires ottomans devaient s'acquitter des droits de douane officiels correspondant à un pourcentage en aspres de deux pour cent sous Mehmed II, pourcentage qui s'est ensuite élevé à quatre, puis cinq pour cent. Istanbul comptait donc parmi les territoires étrangers en matière de droit des douanes. Aussi, est-il certain que ce Kânûnname, tout comme le premier comprenne également des lois plus anciennes. D'ailleurs, Mehmed II l'indique dans son Kânûnname ${ }^{95}$. C'est donc non sans raison que Kraelitz voit une corrélation entre la prise de Constantinople et la structuration progressive des activités de l'Empire ${ }^{96}$, étant donné que l'historienne considère ce Kânûnname comme étant le plus ancien de l'État ottoman. En réalité, aucune preuve n'a pu être obtenue quant à l'existence d'un Kânûnname de ce type à une époque antérieure à Mehmed II. Cependant, il convient de souligner sans plus tarder que les nouveautés apportées par Mehmed II dans le domaine pénal et plus particulièrement dans le domaine fiscal sont moindres au regard de celles qu'il ait pu apporter dans le domaine constitutionnel. Ces nouveautés portent davantage sur l'augmentation des taux d'imposition et sur le mode de collecte des impôts. Quant aux impôts mentionnés dans son Kânûnname, nous sommes en mesure de confirmer leur existence dans la période précédemment citée ${ }^{97}$.

Ce Kânûnname est composé de quatre chapitres : les trois premiers représentent le droit pénal, et le quatrième chapitre rassemble les lois relatives à la fiscalité sous le titre de «Loi de Mehmed Khan ${ }^{98}$ ». En fait, il convient plus exactement de décomposer le 
quatrième chapitre en trois sous-chapitres. Chacune de ces trois lois se conclut par la formule protocolaire "Fait pour considérer ce que de Droit» ou la formule "Fait pour sanctionner ce que de Droit». Ces formules, stipulées sur chaque firman, donnent alors aux lois toute leur force obligatoire par leur nature coutumière. La première loi, à savoir le chapitre premier allant jusqu'à la "Kânûn-i Yürükan (Loi sur les nomades et les militaires) ${ }^{99}$ ", a pour objet la désignation des organismes chargés de collecter l'impôt dont doivent s'acquitter les paysans musulmans de l'Empire vis-à-vis des timariotes au titre des parcelles qu'ils exploitent. En fonction de leur catégorie socioprofessionnelle, ou suivant qu'ils travaillent dans les rizières, ou qu'ils soient pasteurs, comme les Tatars qui ont un statut particulier rattaché à un autre groupe, le pourcentage des impôts dont doivent se dédouaner les sujets ottomans est soumis à des variations.

Le système fiscal dont il est question ici correspond à un système qui se fonde sur la taxe de tenure (çift resmi), propre au régime ottoman. Celui-ci repose entièrement sur le système fiscal coutumier, en concomitance avec les impôts relatifs à la charî'a (l'öşr ou la dîme et la ceziye ou la capitation). Il convient de l'appréhender de la manière suivante : la source de ce système était basée d'une part, sur le système fiscal coutumier pratiqué sur le sol anatolien à l'époque seldjoukide, et d'autre part, sur l'ancien système fiscal mis en place par les autorités de Roumélie que les Ottomans ont chassées à leur arrivée. Sur les terres balkaniques, prévalait le système fiscal byzantin qui était établi depuis des siècles avant l'arrivée des Ottomans, dans lequel les fermiers étaient catégorisés dans différents groupes selon leur état-civil (mariés ou non) et la nature des terres cultivées. L'impôt prenait donc la forme d'un impôt sur la parcelle de terre exploitée (cens - arazi vergisi) et d'un impôt individuel (şahıs vergisi). On peut comparer ces taxes à la «jugum » et la " capito terrena » pratiquées sous l'Empire romain à l'époque du Dominatus. Dans l'optique de clarifier la source du système fiscal coutumier ottoman, nous souhaitons nous arrêter un instant sur ce point qui nous semble important.

Pour ce qui est de ce Kanûnnâme, la taxe de tenure (çift resmi) est décrite de la manière suivante : " Une ferme doit annuellement s'acquitter de trois aspres correspondant à 'la faucille', au 'fléau' et à 'la charrette', elle doit s'acquitter de deux aspres au titre du droit du joug (boyunduruk); pour l'acquittement de ces sept obligations, le montant total dû est de vingt-deux aspres $»^{100}$. Dans un registre du Paşa Sancak (Edirne), rédigé aux alentours de 1456, figuraient les montants correspondant au fermage. Ainsi dans le domaine fermier de Bahadir Bey, on ponctionnait pour chaque çiftlik ${ }^{101}$ (à savoir pour chaque paysan exploitant une tenure de quelques hectares) l'équivalent d'une charrette remplie de bois, une demi-charrette de foin et une charrette de fourrage, ainsi que deux aspres pour l'usufruit de la terre, au titre d'un impôt obligatoire (Hizmet$i$ Reâya).

On s'aperçoit notamment que l'impôt est souvent payé en nature, les produits étant livrés tels quels, sans les transformer en argent. Dans le règlement de Mehmed II, une charrette d'herbe pour le fourrage correspond, donc, à l'acquittement de la "faucille ", tandis que le foin, correspond au paiement du « fléau ». Quant au terme « hizmet » qui y est utilisé, il signifie à la fois "service ", mais dans ce cas précis correspond plutôt au terme «kulluk », servitude, à entendre au sens de «corvée ». Ainsi, les «sept corvées » mentionnées dans l'article correspondant du code coutumier sont les suivantes: en premier lieu, les trois aspres à raison de trois jours de corvées, puis la corvée du bois, qui consiste à transporter une charrette entière de bois, puis la demi-charrette de foin qui correspond à la tâche de battre le blé, et la charrette de fourrage qui correspond à 
la corvée de fauchage; soit trois corvées supplémentaires. Enfin, les deux aspres à payer au titre du droit d'entrée en jouissance de la terre correspondant à la septième corvée. (Cf: art. 2 du même Code "Kanûn-î Cebelüyân » (Loi sur les terres attribuées aux militaires): "Si l'exploitation du beylik comporte un vignoble, ses sujets devront s'acquitter de trois jours de corvées dans les vignes ${ }^{102}$ ", et art. $28:$ "[...] doivent s'acquitter de trois jours de corvées par an à défaut de trois aspres ${ }^{103}$ »). Le service du joug correspond au travail que le paysan fournit avec ses bêtes de somme et sa charrue. On constate par ailleurs que les trois jours de corvées individuelles et le droit d'usufruit sont devenus des taxes payables en pièces d'argent. À ce titre, il est spécifié dans le code de Mehmed II que l'acquittement de ces sept corvées correspond en réalité au paiement de vingtdeux aspres.

Encore une fois dans le code de Mehmed II (art. 28) la mention faite au sujet des artisans du village mérite notre attention : « Les artisans, dans l'obligation de s'acquitter des trois jours de corvées annuelles, ou à défaut, des trois aspres correspondants, ont la possibilité d'être exemptés du travail physique ${ }^{104}$ ». Ici il est montré de manière explicite que les corvées correspondant aux trois aspres exigées consistent à effectuer des travaux individuels physiques. Toutefois les quatre autres corvées exigées ne font l'objet d'aucune dérogation, car elles correspondent aux travaux inhérents à la vie agricole. Chaque fermier (chaque paysan exploitant une parcelle de taille moyenne - de 20 à 60 hectares -) est soumis à cette taxe $\mathrm{e}^{105}$.

Nous sommes d'ailleurs en mesure de rendre compte de l'équivalent en argent des sept corvées, en comparant le règlement organique de Mehmed II et celui de Soliman : une journée de corvée de fauchage ou une charrette de fourrage correspondent à sept aspres, une demi-charrette de foin correspond également à sept aspres et une charrette de bois correspond à trois aspres, soit dix-sept aspres en tout. Si on y ajoute les trois aspres correspondant aux trois jours de corvées obligatoires et deux aspres au titre du droit de joug, on obtient une somme totale de vingt-deux aspres.

Dans certaines régions, le sipahi ${ }^{106}$, ne percevait pas l'impôt sous forme d'argent, au titre de l'exemption de ces corvées, mais prélevait plutôt l'équivalent en nature, à savoir le bois dont il aura besoin pour se chauffer, le fourrage et le foin pour nourrir ses animaux. Toutefois il est clair que si le timar est exploité par de nombreux paysans, le sipahi préfèrera sans doute percevoir cette taxe en argent. D'autre part, il convient d'insister sur le fait que les deux autres formes de corvées - à savoir, les trois jours de corvées obligatoires et la corvée afférente à la taxe d'usufruit -, ont été définitivement supprimées et converties en paiement métallique. Concernant les provinces serbes au début du XVI ${ }^{e}$ siècle, les corvées afférentes au bois, au fourrage et au foin n'avaient pas encore été remplacées par l'acquittement de la taxe en argent. Cette particularité est probablement due à l'incorporation tardive de ce vilayet à l'Empire ottoman (1459).

Quant à la situation des paysans sous l'administration byzantine, on les distinguait tout d'abord selon trois catégories: les Zeugrates, les Boidates et les Aktemones. Selon G. Ostrogorskij ${ }^{107}$, le terme Zeugrate concernait les villageois qui exploitaient une ferme ou un terrain d'une certaine taille ${ }^{108}$. Un villageois exploitant une parcelle d'un rendement de 50 moidoi (unité de mesure byzantine correspondant au boisseau, soit, l'équivalent $d u$ mud ottoman) devait s'acquitter de la somme d'un hyperpère ${ }^{109}$ par an, ce qui au XIV ${ }^{\mathrm{e}}$ siècle équivalait - à peu de choses près - au tarif pratiqué par les ottomans deux siècles plus tard au titre du çift resmi, soit 22 aspres. Les Boidates étaient les paysans qui possédaient des bêtes de somme. Ils payaient tout au plus l'équivalent de la moitié de la 
somme dont devaient s'acquitter les Zeugrates (correspondant à la moitié de l'impôt de fermage ottoman). Quant aux Aktemones, ils représentaient la catégorie des villageois les plus pauvres (l'équivalent ottoman des caba ou kara, extrêmement pauvres et dépourvus de terre à cultiver). En fin de compte, le remplacement des corvées par le paiement d'une somme en argent avait déjà été initié sous l'Empire byzantin. Cet état de choses s'explique aisément par le fait que l'économie monétaire de l'époque avait atteint un degré suffisant de développement.

51 Les Ottomans ont accéléré le processus de modernisation initié à l'ère byzantine ; dans le but de transformer tout le système de corvées en paiement monétaire, ils ont ainsi mis en place un système fiscal savamment structuré. Dans les faits, l'oppression des seigneurs locaux et des monastères à l'encontre des paysans qui se fit grandissante dans les derniers temps de l'Empire byzantin, résulte de ce processus de féodalisation ${ }^{110}$. On peut considérer qu'en s'appropriant les terres et en supprimant la pratique des corvées afin de simplifier le système fiscal, les Ottomans ont introduit de véritables innovations au sein de la paysannerie. De toute façon, le système fiscal ottoman, outre le fait qu'il soit basé sur un système séculaire, s'est probablement perfectionné grâce à sa capacité à adapter à bon escient certains de ses principes ancestraux. $\mathrm{Vu}$ sous cet angle, on comprend mieux pourquoi lors de la conquête des Balkans, les villageois autochtones de confession chrétienne ne se soient pas unis aux instances militaires dans leur lutte contre les envahisseurs ottomans, voire même, qu'ils se soient en quelque sorte ralliés à ceux-ci.

52 La çift resmi (taxe de tenure) déterminant le statut social de la paysannerie ainsi que la nature du système fiscal, est un élément non négligeable dans la mesure où cet impôt a été appliqué uniformément aux musulmans et aux chrétiens des zones conquises, à savoir, l'Anatolie occidentale et la Thrace. En revanche, à l'issue de l'annexion des royaumes slaves, une nouvelle taxe coutumière sera introduite au sein du code juridique ottoman - l'ispence ${ }^{111}$ - s'appliquant uniquement aux populations chrétiennes.

La première partie du Kanûn-i Sultan Mehemmed [Le règlement du Sultan Mehmed] que nous venons d'examiner attentivement concerne les sujets musulmans. Sont mentionnés dans ce texte la çift resmi, puis, après sa mise en place, l'impôt islamique öşr [la dîme], et ses variantes, le $b a c^{112}$, le gerdek ${ }^{113}$, le $u l a k^{114}$ et

le deștbâni ${ }^{115}$ qui diffèrent selon leur mode de collecte et leur montant.

Dans la deuxième partie de la loi figurent, sous le nom de Kanun-i Yürükân, les institutions militaires et les exemptions auxquelles étaient soumis les peuples nomades des Turcomans de Roumélie.

La troisième partie du même code, à savoir, le Kanun-i Cebelüyân bâ Kanûn-i Müzevvec-i Gebrân [loi des soldats et loi sur le mariage des chrétiens], est introduite par une description de l'ispence. Il s'agit d'un impôt individuel qui avait remplacé la çift resmi, dont le montant fixé de longue date à 25 aspres s'appliquait aux hommes chrétiens d'age adulte. Ceux qui payaient l'ispence étaient dispensés du paiement de la çift resmi. Selon le code d'Étienne Douchan ${ }^{116}$, antérieur à l'époque ottomane, un paysan devait travailler deux jours pour le propriétaire d'un pronija - équivalent du timar ottoman - : il devait s'acquitter d'un jour de corvées au vignoble, l'autre journée, étant consacrée aux vignes et au fauchage. En outre, le paysan payait l'hyperpère - monnaie byzantine d'or - une fois par an. Cet impôt annuel est probablement à l'origine de l'ispence. D'ailleurs, dans la 
seconde moitié du XIV ${ }^{e}$ siècle, l'hyperpère représentait en termes de parité de niveau de vie, l'équivalent à peu de choses près, de 25 aspres ottomanes.

Ce chapitre du règlement fait allusion dans son intégralité aux impôts que les sujets chrétiens devaient payer aux propriétaires des timar, à savoir, l'ispence, l'impôt sur le vin et l'élevage des porcs, le droit nuptial (gerdek) d'éventuelles pénalités, le bac, le deştbânî. ${ }^{117}$ La symétrie entre les deux parties de cette réglementation fiscale - la première concernant les musulmans et la seconde concernant chrétiens - tant au niveau de la manière dont elles sont structurées qu'au niveau de leur disposition au sein du texte, est mise en évidence par l'ordre d'énumération des impôts. Il ne fait aucun doute que dans le cas de ce règlement, la systématisation réalisée par Mehmed II est la mieux réussie.

\section{La législation pénale}

Nous avons réservé pour la fin l'analyse de la législation pénale (cerâim kanûnu) qui occupe les trois premiers chapitres du code de Mehmed II, relatif aux sujets de la Sublime Porte. Avant tout, il convient de noter que les dispositions du Code pénal dans l'Empire ottoman se rapportent à une loi unique et universelle, s'appliquant sans distinction à tout l'Empire et à toutes les catégories

L'État ottoman essaya véritablement d'imposer de manière générale certains principes fondamentaux au sein de la réglementation fiscale, telle que l'abolition de la corvée ou de la sujétion personnelle, et les incorpora progressivement à la Kanûn-i Osmanî (Loi ottomane) basée sur la çift resmi (taxe de tenure) et l'ispence (équivalent de la taxe de tenure pour les sujets chrétiens). Par conséquent, une nouvelle réglementation générale ottomane (Kanûnnâme-i Osmanî) fut adoptée en matière de fiscalité. Cependant, l'imposition varie souvent d'une région à l'autre et selon les catégories, qui sont soumises à des statuts juridiques différents, les réglementations fiscales étant régies dans la plupart des cas par les coutumes locales - coutumes représentant généralement les vestiges des législations pratiquées par des États ayant désormais disparu -. Ces lois sont fixées à la suite du recensement des terres (tahrir-i memleket) fraîchement conquises, réalisé in situ par un intendant commissionné par la Porte (tahrir emini). Celles-ci acquièrent leur force exécutoire après avoir été portées à la connaissance du souverain et confirmées par celui-ci. Ce qui explique qu'on prenne d'abord en considération la réglementation propre à chaque sandjak pour ce qui est des questions fiscales. Toutefois, le Code pénal est unique et s'applique à tous sans exception. Cette conception est issue de la prise en considération des peines (ukubât) appliquées en Droit islamique. Les musulmans et les non musulmans sont traités à cet égard de manière semblable. C'est probablement pour cette raison qu'un texte de loi datant de $1583^{118}$ mentionne l'existence d'un « décalage entre la Loi Islamique et le Droit coutumier; certains délits consentis par la charî̀a étant susceptibles de constituer un crime d'une grande gravité au regard de la législation pénale s'appliquant à l'ensemble des territoires de l'Empire $»^{119}$.

Dans la réglementation régissant la province égyptienne ${ }^{120}$, qui faisait alors l'objet de réformes lorsque Süleyman le Législateur, envoya Ibrahim Pacha en 1524 afin d'apaiser l'état d'anarchie qui y régnait, la notion de délit y est évoquée de la manière suivante : " après avoir réclamé l'exemplaire du code en vigueur dans le vilayet Rûm (province grecque) - c'est-à-dire, en Anatolie et en Roumélie -, une copie doit être consignée au Divân 
[Conseil gouvernemental] d'Égypte, une autre copie devant être remise à chacun des cadis et inscrite aux registres correspondants, afin que ces derniers fassent proclamer ce règlement et en ordonnent [l'exécution] dans les Vilayet placés sous leur autorité, en veillant à ce que celui-ci ne fasse l'objet d'aucune altération ou contresens et qu'il ne soit ni violé ni transgressé121. » Il semble donc que le Code pénal imposé en Roumélie et en Anatolie fut diffusé tout aussi officiellement au sein des tribunaux d'Égypte. Un exemplaire de la législation pénale de 1519 produite sous Selim ${ }^{\text {er }}$ et provenant des registres du cadi de Magnisa ${ }^{122}$ est ainsi parvenu jusqu'à nous grâce à ces procédés. On remarque également que "le contenu [des deux codes pénaux] était proclamé de vive voix dans les villes et les villages afin que tout le monde en prenne dûment connaissance et que nul ne puisse l'ignorer ${ }^{123}$ ». Ce caractère universel du Code pénal explique probablement qu'il ne soit envisagé dans le cadre des législations locales propres à chaque sandjak, qu'un « recours à la vieille loi ottomane ${ }^{124}$ » en matière délictuelle ${ }^{125}$.

61 Nous avons actuellement en notre possession les trois premiers codes pénaux ottomans relatifs à la première période, c'est-à-dire, ceux de Mehmed II, de Selim I ${ }^{\text {er }}$ et de Soliman le Magnifique ${ }^{126}$. La comparaison entre ces trois codes révèle que les dispositions du code de Mehmed II sont toujours - à peu de choses près - en vigueur au $\mathrm{XVI}^{\mathrm{e}}$ siècle. Une confrontation du Code pénal d'Alâüddevle ${ }^{127}$ (1479-1515) - dont l'origine était différente de celle des codes ottomans - avec la législation pénale ottomane, démontre du point de vue de leur fondement, l'existence au sein des deux systèmes juridiques, de règlements d'une nature comparable. Ceci nous permet d'émettre l'hypothèse selon laquelle les fondements de la charî'a, ne seraient donc pas les seules sources du droit pénal ottoman, et que les dispositions coutumières auraient également une source commune, qui serait antérieure à la période ottomane.

Le code pénal ottoman reprend les fondements de la charî'a (analogie, prix du sang, etc.). Toutefois, le sultan y introduit, en parallèle, un certain nombre de préceptes d'une portée assez large, notamment au sein des peines de ta'zir ${ }^{128}$ - qui ne sont ni encadrés, ni validés par la Loi islamique -, et ce, en s'appuyant sur le seul principe coutumier. Pour cette raison, les lois pénales ottomanes sont proclamées et rendues publiques sous forme de firmans (décrets), qu'on appelle cerâim-i ma'rufe (délits reconnus par la charî'a) et siyâsetnâme (Traité sur la politique). De même que la proportionnalité des châtiments abordés dans les dispositions de la charî'a - par exemple les passages évoquant le prix du sang à payer pour l'assassinat d'un homme -, fait également l'objet d'un ordre découlant du droit d'initiative sultanien (code de Mehmed II, p. 21, article 2). En raison du fait que les pénalités monétaires constituaient une importante source de revenus alimentant les dirlik ${ }^{129}$ des fonctionnaires tels que le sipahi timariote ou le subaşı responsable des questions d'ordre public, l'État prit conscience de la nécessité d'entreprendre la mise en œuvre d'une réglementation concernant cette question et d'en définir clairement les détails. Par ailleurs, la mise en application des peines relève exclusivement de l'autorité du Sultan. Le cadi n'est habilité qu'à se prononcer sur les décisions relatives à la charî'a ou au droit sultanien. Il est donc naturel que celui-ci se charge directement des lois pénales du sultan. Le souci du maintien de l'ordre public ainsi que la garantie de la sûreté et des intérêts de l'État lui donnent toute latitude à l'expression de sa volonté absolue en la matière. Par conséquent, le domaine pénal constitue un espace juridique ayant bénéficié du développement assez particulier du droit d'initiative sultanien. Sous le règne de Mehmed II, le Sultan proclamait une série de peines coutumières - les peines d'humiliation publique ${ }^{130}$ telles que le rasage forcé de la barbe, la mutilation du nez, la peine capitale ou la confiscation des biens - en 
s'appuyant sur sa propre volonté afin de permettre l'application d'une part importante de décrets-lois et de yasaknâme (code de lois) relatifs aux domaines économique et financier qui seront abordés plus loin. Par exemple, la peine capitale et la confiscation des biens des faux-monnayeurs se convertirent suite à une décision du cadi en une peine d'interdiction de posséder quelconque objet en argent ${ }^{131}$. Par ailleurs, il n'est pas nécessaire de citer les ordres et dispositions discrétionnaires du sultan concernant les peines relatives à des délits politiques. Quant aux peines relatives aux infractions dites politiques, l'évocation du pouvoir absolu du souverain en la matière est ici sans objet.

Décrets-lois [ferman-kanûn]: Lorsque nous avions précédemment mentionné les législations codifiées (müdevven), nous avions signalé que certains articles avaient été empruntés à des décrets-lois qui avaient déjà été adoptés auparavant sur un sujet précis. En fait, les lois coutumières observées sous Mehmed II et avant son règne, se présentaient sous la forme d'ordonnances, proclamées pour résoudre certaines questions, et qui exigeaient à l'époque un agencement correspondant à leur forme primitive. Étant donné que ces ordonnances contenaient des règles de portée générale, elles prirent le nom de kanûn-ferman ou ferman-kanûn (loi-décret ou décret-loi). Même si certaines de ces règles contenaient des articles portant sur un seul aspect d'une question donnée, d'autres, quant à elles, se développèrent sous forme de législations très détaillées incorporant des articles provenant de différentes lois. Par exemple, les décrets relatifs à la frappe de monnaie en argent et aux douanes constituent une législation détaillée de ce type. On peut considérer que les législations codifiées représentent selon toute vraisemblance une forme encore plus détaillée de ce type de réglementations. En réalité nous avons déjà défini plus haut la structure de base des décrets au sein de ces législations.

Le domaine juridique relatif au droit d'initiative sultanien sous le règne de Mehmed II fait l'objet d'une intense activité en termes de production législative, notamment sur le plan économique et financier; d'ailleurs les firman-kanûn atteignent un nombre remarquable, suivant de près le volume des règlements codifiés (sic) produits. Concernant ce genre de document, le texte était consigné et mis en sécurité dans son intégralité ou sous forme résumée dans les registres de mukataa - registres de fermage de la Porte -, dans les registres des cadis et dans les registres de recensement des biens (tahrir); d'ailleurs nous avons publié certains d'entre eux, que nous avons pu retrouver moyennant le passage au peigne fin des registres d'un cadi de Bursa [Brousse] officiant sous Mehmed $\mathrm{II}^{132}$. Il sera véritablement possible de constater et d'apprécier l'étendue de l'activité législative du règne de Mehmed II une fois terminé l'examen minutieux des sources en question se trouvant dans nos archives. Plus d'une cinquantaine de lois du même type, datant de l'époque du souverain Mehmed II furent compilées au sein d'un recueil juridique réalisé à l'époque de Bayezid II ; ce recueil vient d'être publié récemment ${ }^{133}$. Il semblerait que cette compilation de lois ait été mise en place dans le but de rassembler différents exemples de règlements financiers destinés à l'usage du Divân. Il réunit des documents différents, y compris des articles de loi. Ceux-ci se présentent pour la plupart sous forme de brevets (berât), de codes de lois (yasakname), de règlements (kanunnâme) et d'ordonnances (tevki'). Les berât servent à signifier aux fonctionnaires le poste auquel ils sont affectés, et à leur faire prendre connaissance des aspects juridiques et réglementaires se rapportant à leur fonction. En règle générale, les yasakname servent à notifier les lois, dispositions légales et autres protocoles additionnels s'appliquant selon la nature des postes, et enfin les prérogatives accordées aux agents de l'appareil législatif. Les kanûnnâme se présentent quant à eux, 
généralement sous forme de firmans qui servent à informer les fermiers des impôts (mültezim) des lois récemment adoptées dans un domaine donné, dont la force exécutoire, contraint ces mültezim à les appliquer et à les respecter. Enfin, les tevki' sont des ordonnances qui notifient aux hauts dignitaires officiels, tels que le cadi ou le subaş̧ ${ }^{134}$ les nouvelles lois en vigueur. Tous ces décrets se rapportent à la vie économique et financière, - domaines qui ne sont pas pris en compte par la charî'a et comportent pour l'essentiel des dispositions légales (nizâm) relatives aux mines et à ses ouvriers, des lois relatives à la frappe et à la circulation des pièces de monnaie qu'elles soient d'or, d'argent ou de cuivre, des législations en matière de droit des douanes ainsi que des réglementations portant sur la balance des paiements, des réglementations et lois relatives aux monopoles et aux privilèges des produits de première nécessité comme le savon, les céréales et la cire, et enfin des normes relatives au recouvrement de certains revenus de l'État.

Les décrets-lois revêtent souvent le caractère d'un système de gestion administrative qui, dans bien des cas, ne concerne qu'un seul groupe social ${ }^{135}$. Il convient à ce propos, de rappeler le principe du caractère individuel du Droit au sein de l'Empire ottoman, en particulier la dimension locale des lois fiscales et leur application selon chaque groupe. Puisque ces règlements s'adressent directement à une classe déterminée, il est probable qu'ils soient communiqués aux fonctionnaires de l'Administration ottomane sous forme d'ordonnance pour que ceux-ci se chargent de les faire appliquer. Cet aspect juridique inclurait donc les réglementations impliquant les tiers ${ }^{136}$. Les articles de lois qui apparaissent dans ces ordonnances signifient soit une validation des us et coutumes préétablis, soit leur extension à d'autres régions. Par exemple, dans une loi sur les mines proclamée quatre ou cinq ans après l'annexion de Novo Brdo (1455), on voit que celle-ci présente certains éléments juridiques antérieurs à la conquête de Mehmed II, dont les aspects fondamentaux semblent avoir été préservés, voire certains termes étrangers conservés. Parfois, l'exécution d'une règle "selon la loi habituelle » était ordonnée sans même citer les articles de loi correspondants. En réalité "la loi habituelle ", n'est autre que la législation basée sur les us et coutumes. Ce sont en fait des lois qui proviennent d'États pour la plupart déjà disparus mais qui continuent d'exister sous forme d'us et coutumes.

Nombres de ces kanûnnâme et yasaknâme proclamés par Mehmed le Conquérant inspirèrent de nouvelles lois dans les temps qui suivirent. On retrouve au $\mathrm{XVI}^{\mathrm{e}}$ siècle ces mêmes lois telles quelles - bien qu'ayant subi des modifications au demeurant négligeables ${ }^{137}$.

\section{Sur la conception de l'État et du droit sous le règne de Mehmed II}

67 Nous avons jugé pertinent de conclure cet article en rapportant les idées soutenues par l'historien Tursun Bey, - occupant sous le règne de Mehmed II de hautes fonctions au sein de l'État - : celui-ci abordait la question des origines de l'État et du droit, en se basant sur Fârâbî ${ }^{138}$ et Nasîreddîn Tûsî ${ }^{139}$. Ces idées reflètent, dans une certaine mesure, une conception basée sur la supériorité de l'État et du Droit, qui prédominait parmi les cadres de l'administration ottomane à l'époque. Tursun Bey était l'un des intimes confidents du Grand Vizir Mahmud Pacha, un grand homme d'État ayant marqué le 
règne de Mehmed II. Nous nous efforcerons de présenter ci-dessous un résumé des idées qu'il développa au début de sa chronique. ${ }^{140}$ d'ordre divin » (siyaset-i ilâhi). Elle est établie par le Prophète et relève de la charî'a. Si la «mesure » repose uniquement sur le principe de la raison, elle est alors qualifiée de "politique sultanienne et la loi souveraine, que nos spécialistes nomment örf (coutume) ${ }^{141}$ ", à l'instar de la loi de Gengis Khan (Tursun Bey évoque lui-même cette idée). Ces deux types de mesure sont liés à la personne du souverain. On pourrait même affirmer que la première des dites mesures, à savoir, celle régulant la charî'a, est tout de même liée au souverain même si elle n'est pas toujours applicable en l'état. En effet, "une émanation d'ordre divin, telle que la religion musulmane $»^{142}$ est suffisante pour faire régner l'ordre matériel et spirituel dans tout l'univers, jusqu'au jour du Jugement dernier. Toutefois, la présence d'un souverain a de tout temps, relevé de la nécessité absolue. Car sans l'existence du souverain, les hommes ne seraient pas en mesure de vivre comme il se doit, et peut-être s'annihileraient-ils tous les uns les autres, mettant ainsi fin à toute forme d'ordre social.

Dieu impose à tous, petits et grands, la soumission au souverain. Lui seul a droit de vie ou de mort sur ses sujets. Ces pouvoirs attribués au vacib-ül vücud ${ }^{143}$, autrement dit, à l'entité divine, sont également incarnés en la personne du souverain lui-même (autrement dit, le souverain détient l'autorité absolue). En apparence le sultan, en œuvrant à l'aboutissement de ses desseins personnels accroît ses gains et son emprise par le biais de sa fortune et de son armée. Mais son véritable objectif est de répartir ce trésor de la manière la plus optimale par le biais d'une politique appropriée, de faire régner l'ordre grâce à l'armée et de sauvegarder la religion musulmane.

Au sein de tout groupe - même un groupe de brigands - si un individu ne se distingue pas et n'impose ni son autorité ni les ordres qu'il profère, ce groupe est voué à se 
désintégrer. Aussi, «un royaume sans justice ne peut être viable ${ }^{144}$ ». La justice représente donc la condition nécessaire au maintien de l'ordre parmi les Hommes.

Nous avons tenté de résumer ci-dessus les idées de Tursun Bey. Même si l'essentiel de ces idées fut emprunté à Fârâbî et à Nasîreddîn Tusi ${ }^{145}$, certains points sur lesquels Tursun Bey insiste ne manquent de retenir notre attention : l'existence du souverain est indispensable pour la sauvegarde de l'ordre social, ce dernier devant détenir une autorité absolue et sans bornes. Nous avions évoqué au début de cet article, les idées se rattachant à la prééminence de la coutume et du droit dans la conception du souverain quant à la notion d'ordre social.

Naturellement, Tursun Bey souligne le caractère intemporel et omni-inclusif de la charî'a. Cependant, tel que nous avons tenté de l'expliquer précédemment, le droit d'initiative sultanien fit l'objet de très fortes avancées en matière de Droit public, notamment sous Mehmed II tandis que la charî'a, encadrait le droit privé. Il convient à ce titre d'analyser dans le cadre du droit musulman, les deux ouvrages du mollah Hüsrev - autorité reconnue au sein du monde musulman sous Mehmed II - Gurar'ulAhkâm, rédigé en langue arabe, ainsi que le commentaire rédigé en turc, de Durar'ulHükkam, qui servit pendant très longtemps de référence aux tribunaux ottomans.

\section{NOTES}

1. J. Schacht, "The Schools of law and Later Developpments", Law in the Middle East, Eds. M. Khadduri and H. J Liebesney, Washington (1955), p. 73.

2. "Herkim Allahın inzâl ettiği ile hükm etmezse işte kafirler onlardır." [Et ceux qui ne jugent pas d'après ce que Dieu a fait descendre, les voilà les mécréants], Coran, Sourate V (le Festin), verset 44. Traduction française de Muhammad Hamidullah (1909-2002), avec la collaboration de M. Leturmy ,Éd. Amana Publications, août 1989. NDT

3. Cf. H. A.R. Gibb, "Constitutionnal Organization", Law in the Middle East, pp.1-27; E. Tyan, Institutions du Droit Public Musulman, Le Califat, Paris, 1954.

4. Cf. Fuat Köprülü, «Fikh », Islam Ansiklopédisi; J. Schacht, Origins of Muhammedan Jurisprudence, Oxford, 1953.

5. Cf: Ö. L. Barkan, Osmanli Imparatorluğunda Zirâ̂ Ekonominin Hukukî ve Malî Esasları, [Les bases juridiques et fiscales de l'économie agraire sous l'Empire Ottoman], Istanbul, 1942, Index: Örf, örf-i ma'rûf, âdet, örf-i sultanî [coutume, coutume connue, usage, coutume sultanienne].

6. Cf. Reuben Levy, The social structure of Islam, Cambridge, 1957, pp 248-270.

7. Tarih-i Osmanî Encümeni Mecmuas, ilâveler, Tarih-i Ebu'l-Feth [Revue du Comité ottoman d'Histoire, annexes, Histoire du père de la Conquête, de Tursun Beg], p. 13.

8. Levy, The social structure, p. 259.

9. C.S.Hurgonje, Le Droit Musulman, Verspreide Geschriften [Écrits dispersés], vol II, p. 314.

10. Levy, p. 248.

11. Hurgonje, p. 314.

12. Tursun Beğ, Tarih-i Ebu'l-Feth [Histoire du Père de la Conquête], p. 13 ; Ahmedî, Iskendernâme, (éd. N. S. Banarl1), Türkiyat Mecmuası [Revue de Turcologie], vol. VI, p. 111 ; Levy, p. 262.

13. Cf. Makrîzî, Hitat, vol. II, Levy, p. 261. 
14. Grand-vizir d'ascendance ouléma sous Selim $\mathrm{I}^{\mathrm{er}}$ puis sous Soliman le Magnifique. Cf. Robert Mantran (dir.), Histoire de l'Empire Ottoman, Paris, Fayard, 1989, 809 p. NDT

15. Sujet de l'Empire ottoman soumis au paiement d'un certain nombre de taxes. NDT

16. Cf. Gibb, p. 22.

17. Cet ouvrage est rédigé par Muhammed b. Ali b. Süleyman er-Râvendi et porte le titre complet : Râhat'us-Sudûr ve Ayet'üs-Surûr (édit, Ahmed Ateş, Türk Tarih Kurumu, 1999. NDT

18. Texte original : «Imâmın vazifesi hutbe ve dûa ile meşgul olmak... pâdişahliğı (hakimiyeti) sultanlara havâle etmek ve dünyevî saltanatı onların eline bırakmaktır ». NDT

19. Gibb, Ibid.

20. M. A. Köymen, Büyük Selçuklu Imparatorluğu Tarihi [Histoire du Grand Empire Seldjoukide], vol II, Ankara, 1954, pp. 419-459.

21. Cf. F. Köprülü, Islâm Âmme Hukukundan Ayrı Bir Türk Âmme Hukuku yok mudur? [N'existe-t-il pas un droit public turc en dehors du droit public islamique ?], II. Türk Tarih Kurultayı Zabıtları [Actes du II Congrès turc d'Histoire], pp. 383-418.

22. F. Köprülü, Ibid.

23. H. N. Orkun, Eski Türk Yazıtları [Les anciennes inscriptions en turc], vol. I, pp. 28-29, voir à ce propos pp. 34-35, 39, 44, 56 pour comparaison ; S. Maksudi Arsal, Türk Tarihi ve Hukuku [Le Droit et l'Histoire des Turcs], Istanbul, 1947, p. 263.

24. Cuveynî, Tarih-i Cihanguşa'dan naklen (citation tirée de l'ouvrage Histoire de la soumission du Monde), Gibb, pp. 17-18; B. Spuler, Iran Moğolları [Les Mongols d'Iran] traduit par C. Köprülü, Ankara, 1957, p. 408.

25. Al Maqrizi (1364-1442) chroniqueur égyptien. NDT

26. "Şeri'atçı ulema", c'est-à-dire les oulémas ne reconnaissant que la charî'a comme seule et unique loi. NDT

27. Z. V. Togan, op. cit, p. 376.

28. Kadı Burhaneddin (1345-1398) lettré d'origine oghouze, nommé cadi très jeune par le pouvoir Eretnide NDT

29. Iskendernâme, op. cit. p. 111.

30. Cité par Köprülü, à partir de Massignon, op. cit., 414, note $n^{\circ} 67$.

31. Aşık Paşa-zâde, publication de M. Ârif, pp. 19-20. Ebul'ulâ Mardin se trompe en tirant des conclusions trop hâtives concernant ce récit. Aşık Paşa-zâde, par le biais de ce type de récits, reflète le sentiment partagé par une partie de la population qui défend la charî'a, et qui considère les lois coutumières comme facteur de déclin. En effet, le conflit entre la charî'a et l'örf sous Mehmed II éclata ouvertement, et Bâyezid II accéda au trône, en tant que représentant de la défense de la charî'a. Voir à ce propos “Mehmed II”, Islam Ansiklopedisi, fascicule, 73.

32. Ou beylicat dans sa dénomination francisée, qui se rapproche du terme "principauté" : il s'agit d'une subdivision de sancak. NDT.

33. Aşik Paşa-zâde, p. 19. Cet événement revêt une importance capitale, du moins à l'époque du chroniqueur.

34. Orkhan (1281-1362) deuxième sultan ottoman ayant régné de 1324 à 1362. NDT.

35. Z. V. Togan, Moğollar Devrinde Anadolu'nun iktisadî Vaziyeti [La situation économique de l'Anatolie à l'époque mongole], THIM, vol. I, p. 33.

36. Z. V. Togan, Umumî Türk Tarihine giriş [Introduction générale à l'Histoire des Turcs], pp. 330-331.

37. Tapu ve Kadastro Umum Müdürlüğü, Kuyûd-i Kadîme (Direction générale des actes de propriétés et cadastres, archives anciennes].

38. Murâd I ${ }^{\text {er }}$ (règne de 1362 à 1389) : sultan ottoman ayant fondé le premier Beylerbeylik (principauté) et le célèbre corps des Janissaires. NDT.

39. Tahsin Öz, "Murad I. ile Emir Süleyman'a ait iki vakfiyye" [Murad $\mathrm{I}^{\mathrm{er}}$ et les fondations pieuses appartenant à l'Emir Süleyman], Tarih Vesikaları Dergisi [Revue des documents de l'Histoire], $\mathrm{n}^{\circ} 4$ (1957), p. 243. 
40. Déf. du Redhouse, Osmalica- Ingilizce, 17e édition. NDT.

41. Ibid. NDT.

42. Toutes les définitions données ici sont tirées de l'ouvrage Histoire de l'Empire Ottoman, dir. Robert Mantran, Éd Fayard, 1989 NDT.

43. $\mathrm{Cf}$. P. Wittek, $\mathrm{Zu}$ einigen frühosmanischen Urkunden [Concernant certains documents relatifs à la période pré-ottomane], WZKM, vol. 54 (1957), p. 252.

44. Tevârih-i Âl-i Osman [Chroniques de la dynastie ottomane], publication de Fr. Giese, Breslau (1922), pp. 29-34.

45. Mecdî, şakâyık tercümesi [Traduire la pivoine/Traductions Pivoine], Istanbul, (1269 de l'Hégire), pp. 50. 100, 1, 112.

46. La création du système du devşirme, mode de recrutement des esclaves chrétiens, qui une fois formés, sont destinés à incorporer le rang des janissaires ou assumer de hautes fonctions au sein de l'Etat remonte à Bâyezid I ${ }^{\mathrm{er}}$ NDT.

47. Qui signifie "le faux Mustafa", celui-ci s'est fait passer pour le fils de Bâyezid I ${ }^{\text {er }}$ (tenu pour mort au champ de bataille), s'étant ainsi ménagé des alliances stratégiques notamment dans l'Empire byzantin, pour déstabiliser Mehmed I ${ }^{\mathrm{er}}$ (1413-1421) NDT.

48. Seyh Bedreddin Mahmud (1359-1420) homme de foi révolutionnaire et prédicateur charismatique, il a pris la tête d'un mouvement de rébellion contre l'Empire ottoman en 1416. NDT.

49. F. Köprülü, ş. Yaltkaya, Fr. Babinger et enfin Kissling qui ont examiné ces rébellions n'ont pas assez insisté sur leur lien avec les uç (marches frontalières) et le problème des timars. Voir l'article suivant: "Ankara bozgunundan Istanbul'un zaptına" (De la défaite d'Ankara à la prise d'Istanbul), Belleten, $\mathrm{n}^{\circ} 27,(1943)$.

50. Terre dont le revenu est attribué à un timariote, militaire, ou administrateur civil NDT.

51. Sûret-i Defter-i Sancak-i Arnavid (Structure du registre du Sandjak d'Albanie), publication d'H. Inalcik, Ankara, (1954).

52. Cf. Ibid., Introduction, p. 23.

53. Türk Hukuk ve Iktisat Tarihi Mecmuası (Revue sur l'Histoire du Droit et de l'Économie turcs), vol I, pp. 165-298.

54. Cf. "Mehmed II", Islam Ansiklopedisi, fascicule, 73.

55. Tevârih-i Âl-Osman (Chroniques ottomanes) $\mathrm{I}^{\mathrm{er}}$ cahier (manuscrits), Introduction. Millet Kütüphanesi (Bibliothèque Millet). A. E. 25.

56. Règlement organique NDT.

57. Köprülü, article cité, p. 410.

58. Cf. Spuler, à propos de la hiérarchisation des règlements organiques d'après des données sur les Mongols d'Iran, op. cit, pp. 409-411.

59. Fr. Kraelitz (Mitteilungen des Osm. Gesch. I) et M. Arif, TOEM.

60. Ö. L. Barkan, Osmanl. Imparatorluğunun..., Introduction, pp. 15- 24.

61. Palais de Topkapi d'Istanbul, Revan Köşkü kitapları [Livres du kiosque Revan], nº 1926.

62. Subdivision d'une province (Vilayet). NDT.

63. Texte original : "Paşa Hazretlerı dahi anınla cümle memâlik hususâtın bu kanûn-i şerîf muktezasinca amel ede » NDT.

64. Texte original : "Yeni ve mûteber kanûnnâme" NDT.

65. Ahmed I ${ }^{\text {er }}$ (règne 1603-1617) sultan ottoman NDT.

66. Ç. Uluçay, Saruhan'da Eşkıyalık (Les Maquis de Sarukhan), Istanbul, 1944, p. 164.

67. Ou «Soliman le Législateur » un des surnoms donnés à Soliman le Magnifique (1520-1566) en raison de l'importance de ses contributions dans le domaine juridique NDT.

68. Cf. Atti del X. Congresso Internazionale, Comitato Internationale di Scienze Storiche [Actes du Xe Congrès International, Comité International des sciences historiques], Rome, (1957), p. 393.

71. Préambule au Kanûnnâme rédigé par M. Ârif. p. 18. 
72. Haut dignitaire religieux de l'Islam, il règle en dernier ressort les points de controverse de la charî'a.

73. L'institution du Nişancllk est vraisemblablement antérieure à Mehmed II. L'existence du Nişancı Ibrahim Pacha en 1444 est selon nous avérée. Cf : H. Inalcik, Fatih Devri I. [La période de Fatih Tome I], Ankara (1954), p. 87.

74. Kanûnnâme, TOEM, suppléments, p. 23.

75. Texte original : «Ahval-i saltanata nizâm ». NDT.

76. Terme d'origine persane signifiant "à l'intérieur" désignant la partie privative du Palais, qui représente néanmoins un poids non négligeable dans la direction que peut prendre la gestion de l'État, par opposition au Biroun (signifie « extérieur » en persan) qui désigne la partie du Palais destinée à la tenue des auditions publiques, des réceptions officielles. NDT.

77. Levée des esclaves. Système méritocratique propre à l'administration ottomane, qui consistait à fournir une éducation privilégiée aux esclaves se démarquant par des aptitudes ou talents particuliers. Les plus doués pouvaient être amenés à assumer les plus hautes fonctions de l'État, comme celle de grand vizir NDT.

78. Décrets impériaux NDT.

79. Texte original : "ebed'ül âbâd ma'mûlün bih". NDT.

80. Texte original : benim dahi kanûnumdur". NDT.

81. Fonction du Chef de l'Administration financière de l'Empire.

82. Fonction du Nişanc1.

83. Les établissements d'enseignement supérieur situés dans la cour de la Mosquée Fatih d'Istanbul. NDT.

84. Grand Hall situé dans un édifice public, où se tenaient les audiences des hauts dignitaires de l'Empire ottoman ou des ambassadeurs avec le sultan. NDT.

85. Texte original: "Cenâb-i şerîfimle kimesne taâm yemek kanûnum değildir, meğer ehl-i iyâldan ola. Ecdâd-i izâmım vüzerâsile yerlerimiş, ben ref' etmişmdir.". NDT.

86. Cf. H. Inalcık, Fatih Devri, I, Zeyil no. VII, s.215, et H. Inalcık, Sûret-i Defter-i Sancak-i Arvanid, Giriş [Introduction].

87. Royaume ennemi des Ottomans à l'époque de Murad II. NDT.

88. Bizans Tarihi [Histoire de Byzance], traduction de V. Mırmıroğlu, Istanbul 1953, p. 113.

89. Hauts dignitaires religieux de l'Islam. NDT.

90. Ce terme signifiant "garçon » désigne les jeunes serviteurs des appartements privés du sultan .NDT.

91. Magistrats ottomans dont l'autorité s'exerce dans le cadre de la loi canonique islamique. NDT.

92. À propos du droit administratif sous Mehmed II, il existe des sources turques et italiennes contemporaines à cette époque d'une importance capitale, qui permettent de compléter les informations fournies par les sources officielles. La première source concerne les informations apportées par Idris Bitlisi, dans son Heşt Behişt [Huits paradis] sur le droit administratif à l'époque de Mehmed II, qui sont de première main et très complètes. L'œuvre de Promontorio De Campis, rédigée en 1475, au sujet du droit administratif de la période ottomane ainsi que celle de JeanMaria Angiolello, qui avait pendant de longues années séjourné dans le Palais impérial ottoman en tant que page servant dans les appartements privés du sultan, s'étant ensuite échappé en Italie où il rédigea une "Histoire des Turcs", sont sans doutes les sources italiennes qui comptent parmi les plus importantes. La seconde source a été publiée par Franz Babinger : Bayer. Academie der Wissen., Phil.-Hist. Klasse, Sitzsungb.1957, heft 8, München 1957. Quant à Angiolello, il a été publié en 1911 à Bucarest par I. Ursu. En comparant ces sources avec les kanûnnâme et les supports divers et variés dont nous disposons dans nos archives, l'organisation de l'Empire ottoman peut être décrite de manière parfaite, mais ceci n'a pas encore été fait à ce jour.

93. Ou Kazasker, Juge Militaire Suprême. NDT. 
94. Mitteilungen zur osmanischen Geschichte [Introduction à l'Histoire de l'Empire ottoman], vol. I (1922), pp. 13-48.

95. Ibidem p. 9, article 6 .

96. Einleintung, p. 18.

97. Voir : Sûret-i Defter-i Sancak-i Arvanid [Copie du Registre de la province albanaise], pp. XXVIIXXXVI.

98. Texte original: "Kanûn-i Sultan Mehmed Han". NDT.

99. Ibid. pp. 23-25.

100. Ibid. p. 23.

101. Exploitation agricole d'une surface relativement importante subdivisée en plusieurs terrains (çiftlik) cédés à plusieurs paysans au titre du fermage. NDT.

102. Texte original : "Beğlik bağa varsa raiyyetleri o beğlik bağa yılla üç gün işlede”. NDT.

103. Texte original : "Yilda üç hizmet veya üç akça vere". NDT.

104. Texte original: "Yılda üç hizmet veya üç akça vere, ziyade sanat ehlidir deyüp gücile iş işletmiyeler". NDT.

105. Cf. H. Inalcik, Le règne de Fatih, tome I, p. 171.

106. Cavalier qui se voyait accorder un timar en échange de services obligatoires, dont les prélèvements fiscaux générés représentent la solde. NDT.

107. Historien soviétique éminent spécialiste du Monde Byzantin. NDT.

108. G. Ostrogorkij, pour l'histoire de la Féodalité Byzantine, Bruxelles (1954), p. 297.

109. Ou Hyperpérion, monnaie Byzantine sous forme de pièce d'or, apparue pendant le MoyenAge. Source. Cf. Richards, John F. (1983). Precious metals in the later medieval and early modern worlds. Durham: Carolina Academic Press, 1983, pp. 57-59. NDT.

110. G. Ostrogorskij, op. cit., pp. 155-180.

111. Ou Ispenç: impôt annuel de vingt-cinq aspres versé par les paysans chrétiens (au lieu de la çift resmi).

112. Taxe des transactions commerciales intra-muros NDT.

113. Ou Resmî Arusâne : équivalent du Droit nuptial ; taxe dont doit s'acquitter une jeune fille se mariant. Cf. Coşkun Üçok, (1944). Osmanlı Devleti Teşkilatından Tımarlar, AÜHF Dergisi, I/4 Ankara, pp. 525-551 NDT.

114. "Taxe des relais postaux»: tout type de documents, messages et correspondances à caractère officiel étaient transportés par des messagers de la Porte, qui utilisaient des aires spécialisées - sortes de relais permettant aux messagers de se reposer, de ravitailler leurs chevaux, etc.- qui jalonnaient les routes empruntées par ceux-ci. Les habitants vivant à proximité de ces relais étaient soumis à une taxe dite «ulak» (taxe des relais postaux), en conséquence des retombées positives (au niveau de la sécurité assurée par la présence des autorités, propice à la prospérité et au développement du commerce) dont ceux-ci bénéficiaient. Cf. : Aryeh Shmuelevitz, The jews in the Ottoman Empire in the late fiftieth and sixteenth centuries, Brill archives, (1984), 207pp, p96 NDT.

115. Honoraires pour le gardien des pâturages. (c.f. Metin Coşgel, dir.) ; “A glossary of terms used in ottoman tax registers"; Histoire économique de l'Empire Ottoman, Dpt des sciences, Université de Connecticut. Disponible en ligne sur: http//www.ottoman.uconn.edu/Glossary/ Tahrir_glossary.htm NDT.

116. Étienne Douchan (1308-1355), surnommé le Fort, prince des Serbes. Sous son règne, la Serbie atteint son apogée territoriale et devient l'un des plus grands États d'Europe. En dehors des conquêtes, il fait appliquer un système universel de lois sous le nom de Code Douchan. (Cf. Lazaire Douma ; "Étienne Douchan » ; in Marcellin Berthelot, Camille Dreyfus (et al.) ; La grande encyclopédie ; H. Lamirault et Cic éditeurs, Paris, 1885-1902 ; vol. 16, p. 658.). NDT. 
117. Article 6, « harac-güzârlardan » au lieu de « harac görenlerden » comme Kraelitz le lit. En fait, sa lecture et sa traduction vers l'allemand contient beaucoup d'erreurs. Ö. L. Barkan ne put pas les corriger, car il suivait la publication de Kraelitz (cf. Ömer Lufti Barkan, op. cit.; pp. 387-395).

118. Yeni-Il kanunnamesi [code de Yeni-il]. (cf. Ömer Lufti Barkan, op. cit. ; p. 81).

119. Texte original : “âmme-i memâlik-i mahrûsada cinâyât mukabelesinde vaz' olunan cerâim-i ma'rûfe" NDT.

120. Cf. Barkan op. cit., p 362.

121. Texte original: "Rûm vilâyetinde icra olunan kanûnnâmenin sûreti talep olunup dahi bir sûreti Misır divânında hıfz olunub ve birer sûreti dahi her kadıya göderilib sicillâtına sebt olunduktan sonra taht-i kazâsında olan vilâyete nidâ ve tenbîh ettire ki, bu kanûna mugâyir ve muhâlif nesne alınmayıp ta'addî ve tecavüz olunmaya." NDT.

122. Ce siyâsetnâme [Traité sur la politique] trouvé par Ç. Uluçay fut publié dans Belleten (no 21-22) par E. Z. Karal. Le droit pénal (ceza hukuku) islamique-ottoman est traité dans son intégralité par Coşkun Üçok dans Ankara Hukuk Fakültesi Dergisi [Revue de la faculté du Droit d'Ankara], n III.

123. Texte original : "Şehirlerde ve kasabalarda mazmûnu nidâ olunup, âmme-i nâsa i'lam ve i'lan" NDT. 124. Texte original: "kanûn-i kadîm-i Osmanîye müracaat olunması" NDT.

125. Comme on peut l'observer par exemple dans les règlements que Barkan publia: Boz-Ulus (1540), p. 143 ; Malatya (1559), p. 117 ; Hatvan (Hongrie) relatifs à l'époque de Mehmed IV, p. 317.

126. Code de Fatih, pp. 19-23 ; Siyasetname de Selim I, Belleten, no 21-22 ; Code de Soliman, Târih-i Osmanî Encümeni Mecmuası (TOEM), en annexe, pp. 1-10.

127. Fils de Süleyman Bey, appartient à la dynastie des Dulkadir, turkmènes d'Anatolie. Il se rebelle en 1472 sous le règne de Mehmet II et arrache le pouvoir à son frère şahbudak Bey. (c.f. «Alâüddevle bin Süleyman, Bozkurt », Yılmaz Öztuna (dir.), Türk ansiklopedisi [encyclopédie turque] ; Ankara, Milli Eğitim, 1943-1985 ; vol. 2, p. 7.) NDT.

128. Terme du Droit pénal musulman qui désigne les peines qui dépendent des réglementations locales et juridictions compétentes NDT.

129. Rémunération versée à un fonctionnaire de la Porte au titre des services rendus NDT.

130. Robert Anhegger, Halil Inalcık, Kanûnnâme-i Sultanî ber Müceb-i Örf-i Osmanî, Ankara, TIK, (1956); document $\mathrm{n}^{\circ} 22$.

131. Ibid. documents $2,5,8$.

132. Belleten, $\mathrm{n}^{\circ} 44$ (1947), pp. 697-703.

133. Anhegger, Halil Inalcık, op. cit.

134. Le subaşı a les mêmes attributions que le Sancak Bey, mais à un niveau moindre, le subaşıllk [Subdivision de sandjak]. Ils assurent l'application des lois et la présence de l'État jusqu'aux confins de l'Empire NDT.

135. Cf. à titre d'exemple : maden yasaknâmeleri [Les yasakname sur les mines], Robert Anhegger et Halil Inalcik, op. cit., pp. 5-13.

136. Cf. à titre d'exemple, "gümüş yasaknâmeleri [les yasakname sur l'argent (matière)]»; ibid.; pp. 3-5.

137. Cf. Tursun Bey; op. cit.

138. Al Farabî (872-950) né en Transoxiane : grand philosophe hellénisant musulman de langue arabe, connu en occident sous les noms d'Avennasar ou Alfarabius NDT.

139. Nasîreddîn Tûsî (1201-1274), savant persan originaire de Tus. Il s'est illustré par ses contributions à la science, la philosophie, l'astronomie, la théologie, aux mathématiques et à la médecine NDT.

140. Tarih-i Osmanî Encümeni Mecmuasl, annexes, p. 11-13.

141. Texte original : "siyâset-i sultâni ve yasag-i padişahî derler ki, urefâmızca ona örf derler" NDT.

142. Texte original : "vaz'-i ilahî meselâ dîn-i islâm" NDT.

143. "Celui dont l'existence est nécessaire, Dieu » NDT.

144. Texte original: "mülk-i bî adl pâyidâr olmaz" NDT. 
145. On retrouve ces idées fondamentales, empruntées de la philosophie hellène, dans Siyasat'ul Madina et Arâ'u ahl'ul-madinat'il fâdıla, Farâbi, fils d'Uzluk, et dans Ahlak-i Nasırî, de Nasîreddin Tûsi. Cf. Dwight M. Donaldson, Studies in Muslim Ethics, Londres, SPCK ; 1953, 332 p.

\section{RÉSUMÉS}

Un système juridique basé sur la coutume et la volonté du sultan complète à partir du XIV siècle la charî'a, affirmant la force de la tradition turco-mongole.

En dépit du caractère religieux de l'Empire ottoman, un système juridique séculaire basé sur la coutume et établi par la seule volonté du souverain se développe notamment à partir du XIV siècle, s'octroyant une place significative aux côtés de la jurisprudence islamique. Les lois relatives à ce droit coutumier, rentrant parfois en conflit avec la charî'a, sont d'une certaine façon liées à la tradition turco-mongole, constituant ainsi une preuve incontestable de la place absolue et centrale qu'occupe le souverain dans la conception de l'Etat au sein des organisations turco-islamiques.

Despite the religious nature of the Ottoman Empire, a secular legal system based upon social customs, established by the sovereign, developed mainly from the $14^{\text {th }}$ century, eventually taking a place of significant importance alongside Islamic Law. The rules relating to this kind of law, that may in some cases contradict the principles of the Chari'a-, are somehow associated with Turkish and Mongol traditions, therefore constituting undeniable evidence asserting the absolute and central position of the sovereign in Turkish-Islamic organization's traditional conception of the state.

\section{INDEX}

Index chronologique : Empire ottoman

motsclesmk ОТОМАНСКАТА ИМПЕРИЈА

motsclestr Osmanlı İmparatorluğu

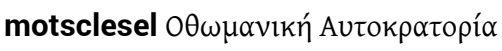

Mots-clés : aspre, Çift resmi, droit coutumier, Fatwa, législation ottomane, Mehmed II, Ibn Khaldûn (1332-1406), Kadiaskerlik, pouvoirs du sultan, Örf, charî'a, Berat, Divan, Firman, Ispence, Cadi/Kadi, Kanunname, Mufti, Mukataa, Mültezim, Nişancı, Oulema, Raïa/Raya/Reaya, Sandjak, Sipahi, Timar, Vakif, Vilayet, Umma, Mehmed II

Thèmes : Histoire

Keywords : ottoman legislation, customary law, chari'a, Mehmed II, turk-mongol tradition, Ottoman empire, History 